\title{
Host-guest binding in water, salty water, and biofluids: general lessons for synthetic, bio-targeted molecular recognition
}

\author{
Meagan A. Beatty and Fraser Hof* \\ Department of Chemistry, University of Victoria, 3800 Finnerty Rd, Victoria, BC, V8W 3V6 \\ Canada
}

*fhof@uvic.ca

\begin{abstract}
Synthetic molecular recognition systems are increasingly being used to solve applied problems in the life sciences, and bio-targeted host-guest chemistry has rapidly arisen as a major field of fundamental research. This tutorial review presents a set of fundamental lessons on how host-guest molecular recognition can be programmed in water. The review uses informative examples of aqueous host-guest chemistry organized around generalizable themes and lessons, building towards lessons focused on molecular recognition in salty solutions and biological fluids. It includes selected examples of macrocyclic host systems that work well, as well as common pitfalls and how to avoid them. The review closes with a survey of the most important recent advances, which involve host-guest chemistry in living cells and organisms.
\end{abstract}

\section{Introduction}

Supramolecular chemistry, or chemistry beyond the molecule, involves the design and study of simple components interacting through non-covalent bonds to form larger, more complex assemblies. Nature has mastered the art of directing intricate molecular assemblies in water, yet supramolecular chemists are still learning the rules. Within a cell, many types of assemblies have evolved to carry out the functional requirements of life. All such assemblies need to form in aqueous solutions that contain various salts, competitive co-solutes, and other proteins. Chemists arguably have access to a much wider array of molecular building blocks, functional groups, and molecular properties than those routinely used by Nature. However, it remains a major challenge to create synthetic molecules that are soluble in water and can still carry out their programmed binding function in the presence of salts and co-solutes. 
One goal that motivates much of supramolecular chemistry is to mimic Nature's sophisticated assemblies, while adding unique characteristics that chemists can program into the system. Although there are many strategies to develop such assemblies, one common strategy is to synthesize a concave 'host' molecule that contains a recognition motif for a specific secondary 'guest' molecule. Upon recognition of the two components, a complex is formed based on the strength of non-covalent bonds that are shared between each component. The medium that solvates each component influences the strength of the non-covalent bonds and in turn the complex. Early supramolecular systems were almost exclusively studied in organic solvents. Yet mimicking Nature requires systems to operate in water, which interacts with the assembling components much differently than organic solvents. Computational and supramolecular chemists have collaborated to model binding interactions through the SAMPL (Statistical Assessment of the Modelling of Protein and Ligand) host-guest blind challenge exercises (https://github.com/samplchallenges). These have tracked the major improvements in the field, but have also shown that we are still not able to fully understand or predict host-guest chemistry in water - especially where salts are involved. Chemists have reported many systems that mimic different aspects of natural molecular recognition and self-assembly, and from this history we can identify key lessons.

Creating a host that predictably binds guests in water, salty water, or biological fluids remains a major challenge. This tutorial review is aimed at researchers who are starting in the area of aqueous host-guest chemistry. The lists of non-covalent interactions that are used to program molecular recognition of guests (e.g. hydrogen bonds, cation- $\pi$ interactions, dispersion forces, electrostatics, the hydrophobic effect, and more) will be familiar to our target audience, and are well described in textbooks. ${ }^{1}$ These will be alluded to without detailed descriptions, except where necessary for the specific lessons within this tutorial. Many comprehensive reviews of aqueous molecular recognition have also been presented elsewhere. One specific goal of this tutorial review is to present strategies that are used to create hosts that work in water, and to derive general lessons about those strategies. Another goal is to teach researchers who are new to the field about the common challenges, and how to achieve success in programming their own host-guest systems for operation in salty aqueous solutions. Our approach starts with introducing the key concepts and strategies in aqueous host-guest chemistry, showing illustrative examples, highlighting common pitfalls, and presenting general lessons. In order to show the most important recent advances, we 
will finish with a section covering the relatively small number of host-guest systems that successfully function in real biofluids and living systems.

\section{An introduction to the common building blocks}

We will focus mainly on an informative subset of macrocyclic hosts, including cyclophanes, cucurbiturils, and cyclodextrins. Cyclophanes are macrocycles consisting of an aromatic repeating unit connected by a bridging component. A major branch of cyclophanes that has produced many lessons in molecular recognition is the family known as calix[n]arenes (Figure 1a); the calix[4]arene is comprised of phenol units repeated $n=4$ times and bridged by $n=4$ methylenes. ${ }^{2}$ Pillar[n]arenes are relatives that involve [n] hydroquinone units similarly bridged by [n] methylenes (Figure 1b). Other cyclophanes will be introduced as they arise in the review. To solidify key lessons and highlight important comparisons, some non-cyclophane hosts such as cucurbiturils and cyclodextrins will also be included. Briefly, cucurbiturils (CBs) are rigid hosts that contain glycoluril units bridged by pairs of methylenes, with the most common members being CB5-CB8 (Figure 2a-b). Cucurbit[n]urils feature a hydrophobic cavity and two symmetric portals lined by carbonyl oxygen atoms. Cyclodextrins (CDs) are cyclic oligosaccharides that contain a repeating glucose molecule joined by a glycosidic bond, in which the rims and exterior of the toroid are lined with hydroxyl groups, and the cavity is lined with hydrophobic $\mathrm{CH}$ groups. As the number of glucose units varies from $6-8$, the cyclodextrins are called $\boldsymbol{\alpha}-\mathbf{C D}, \boldsymbol{\beta}-\mathbf{C D}$, and $\boldsymbol{\gamma}-\mathbf{C D}$, respectively (Figure 2c-d).

a)

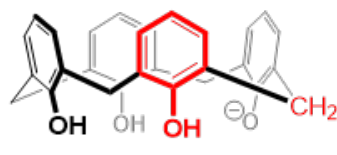

Repeating units, $\mathrm{n}=4$

Calix[4]arene b)

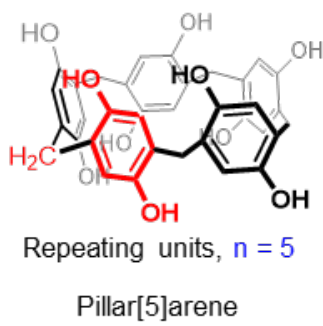

Figure 1. Examples of cyclophane type hosts. (a) Calix[4]arene and (b) pillar[5]arene involve repeating aromatic and methylene subunits that each enclose a guest-binding cavity. 
a)

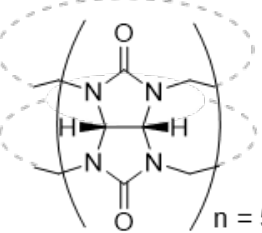

c)

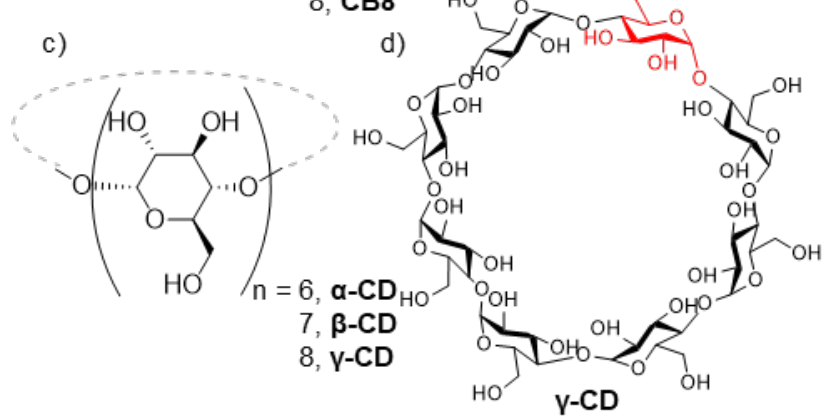

b)

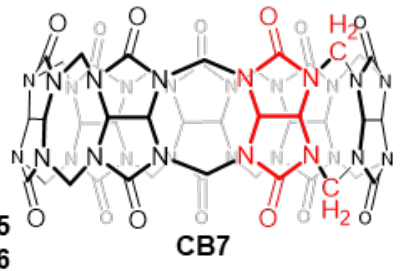

6, CB6

7, CB7

8, CB8

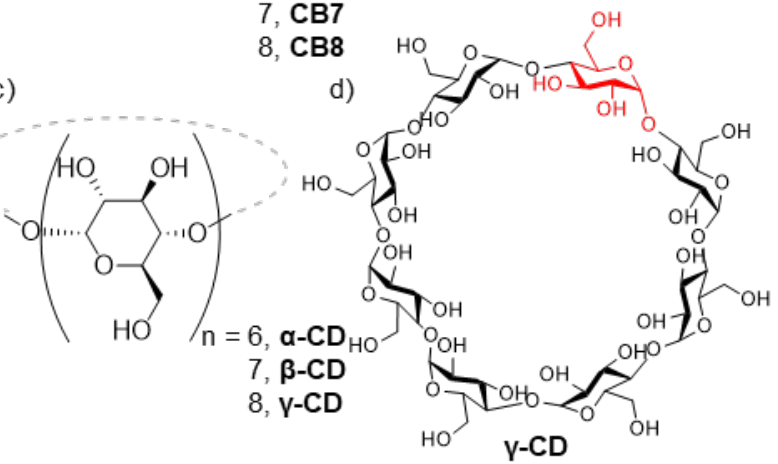

Figure 2. Cucurbiturils and cyclodextrins are macrocyclic hosts that make notable host-guest complexes. a) Cucurbiturils vary by the number of glycoluril units. b) CB7 is a commonly used cucurbituril. c) Cyclodextrins are formed from repeating units of glucose. d) An example is $\gamma$ CD.

\section{Strategies for introducing water solubility}

The first step toward making organic hosts functional in an aqueous medium is to ensure they are water soluble. Early work with cyclodextrins, which are inherently water soluble due to their external-facing hydroxyl groups, demonstrated that hydrophobic cavities provide strong binding of organic guests in aqueous solutions. ${ }^{3}$ Cyclophanes became interesting targets as they also provide hydrophobic pockets and can be synthetically manipulated to form diverse recognition sites. Many cyclophane derivatives have been synthesized with varying charged functional groups that render them both water-soluble and efficient at binding guests in water.
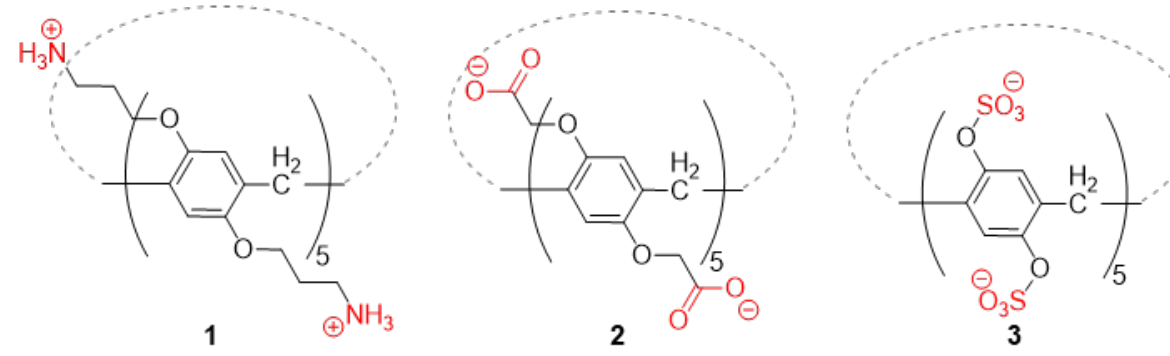

Figure 3. Pillar[5]arenes show the varied effects of being functionalized with different charged groups. Oppositely charged pillar[5]arenes bearing ammonium (1) and carboxylate groups (2) 
prefer guests with different charges, and when mixed with each other form bilayers due to electrostatic interactions between the two kinds of hosts. Pillar[5]arene bearing sulfates (3) binds diammonium ions much more strongly than does $\mathbf{2}$, in spite of their similar charges.

A large diversity of charged groups are routinely used. Sulfonates, carboxylates, and phosphonates are commonly employed to create negatively charged hosts. Ammonium ions, guanidinium ions, and pyridinium ions are frequently used to create cationic hosts. Some predictable effects arise: the pillar[5]arene host with ammonium ions (1, Figure 3) binds a variety of carboxylate containing guests; the analog with carboxylates (2) binds a variety of cationic guests; a mixture of $\mathbf{1}$ and $\mathbf{2}$ leads to aggregates of alternating cationic and anionic host molecules. ${ }^{4}$

Not all similarly charged groups are created equal. Hosts bearing multiple groups such as carboxylates, which have $\mathrm{pKa}$ values relatively close to the $\mathrm{pH}$ at which the host is operating, don't always remain fully charged under experimental conditions. Hosts with either permanently fixed charges (e.g. quaternary ammonium ions) or more extreme $\mathrm{pK}_{\mathrm{a}}$ values (e.g. sulfonates or sulfates) are more likely to stay charged, soluble, and functional under all solution $\mathrm{pH}$ values. Pillar[5]arenes with pendant sulfate (3) groups provide an example of how a subtle change in group can produce large effects (Figure 3). ${ }^{5}$ The sulfate 3 is directly attached to the macrocycle which creates a very large, local buildup of charge that is directly lining the binding pocket. Although the number of potential anionic groups is the same in $\mathbf{2}$ and $\mathbf{3}$, the potency of binding for diammonium ions is orders of magnitude higher (up to 88000 -fold) for $\mathbf{3}$. This can be attributed to the flexibility of the linkages to carboxylates, which allow them to partially collapse onto one another, as well as the very low $\mathrm{pK}_{\mathrm{a}}$ of sulfates, which ensures they all remain anionic in spite of their close proximity to each other.

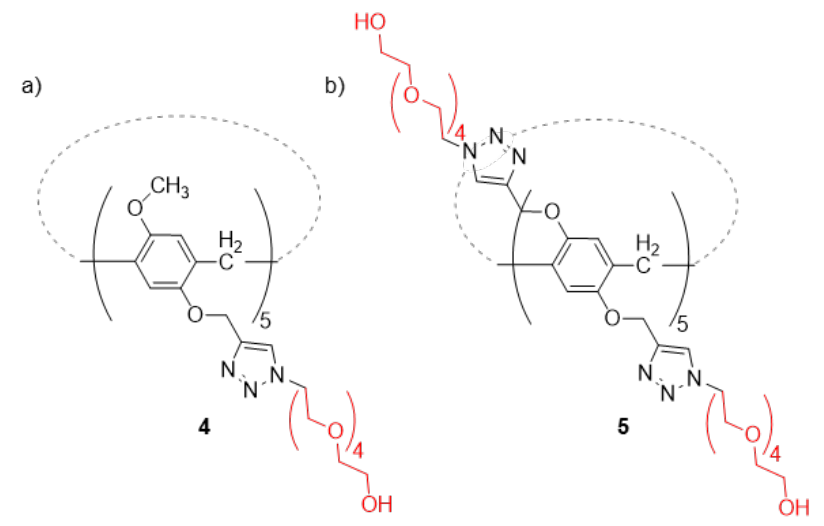

Figure 4. Polyethylene glycol tails improve water solubility without introducing electrostatic interactions from charged functional groups. a) A single glycol tail improves solubility ( $1 \mathrm{mM}$ with $10 \%$ DMSO) while b) two glycol tails renders the host completely water soluble (1 mM). 
a)

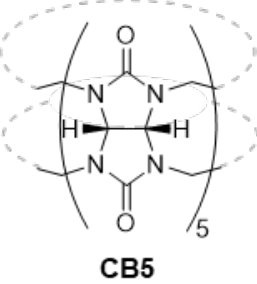

c)

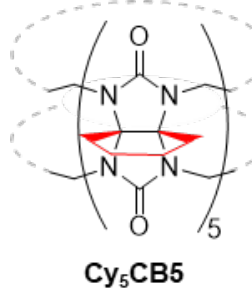

b)

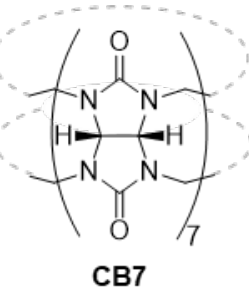

d)

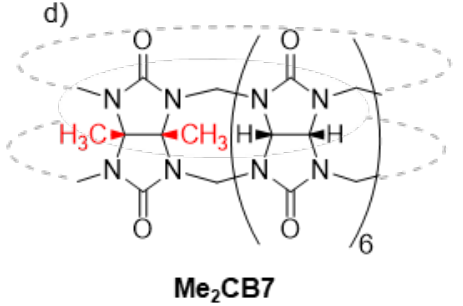

Figure 5. Structurally modifying cucurbiturils improves their water solubility. a) CB5 and b) CB7 are moderately water-soluble $(20-30 \mathrm{mM})$. Analogs substituted with neutral groups such as c) Cyclohexyl-CB5 $\left(\mathbf{C y}_{5} \mathbf{C B 5}\right)$ and d) dimethyl-CB7 $\left(\mathbf{M e}_{2} \mathbf{C B} 7\right)$ can have improved water solubility $(\geq 200 \mathrm{mM})$.

Neutral polar functional groups are an alternate strategy to increase water solubility. For example, when polyethylene glycol tails have been appended to one rim of pillar[5]arenes (4, Figure 4) they become water-soluble with the aid of $10 \%$ DMSO. ${ }^{6}$ When both rims display polyethene glycol tails (5) then the host is fully soluble in water without the aid of any organic solvent. Cucurbiturils contain neutral polar carbonyls, but many of these hosts are only moderately water soluble (20-30 mM for CB5 and CB7, Figure 5a and b). ${ }^{7}$ Although synthetically modifying these hosts is not trivial, there exist CBs externally functionalized with neutral alkyl groups that are more water soluble than their unfunctionalized counterparts. For example, cyclohexyl-CB5 (Cy ${ }_{5}$ CB5) is water soluble up to $\sim 200 \mathrm{mM}$, while the addition of two methyl groups on CB7 (Me 2 CB7) increases its water solubility to $264 \mathrm{mM}$ (Figure 5c, d). ${ }^{7,8}$ These results can't be easily understood, because the new groups added are themselves nonpolar, but their ability to increase water solubility presumably comes from some form of perturbation of stable packing in the solid state. 


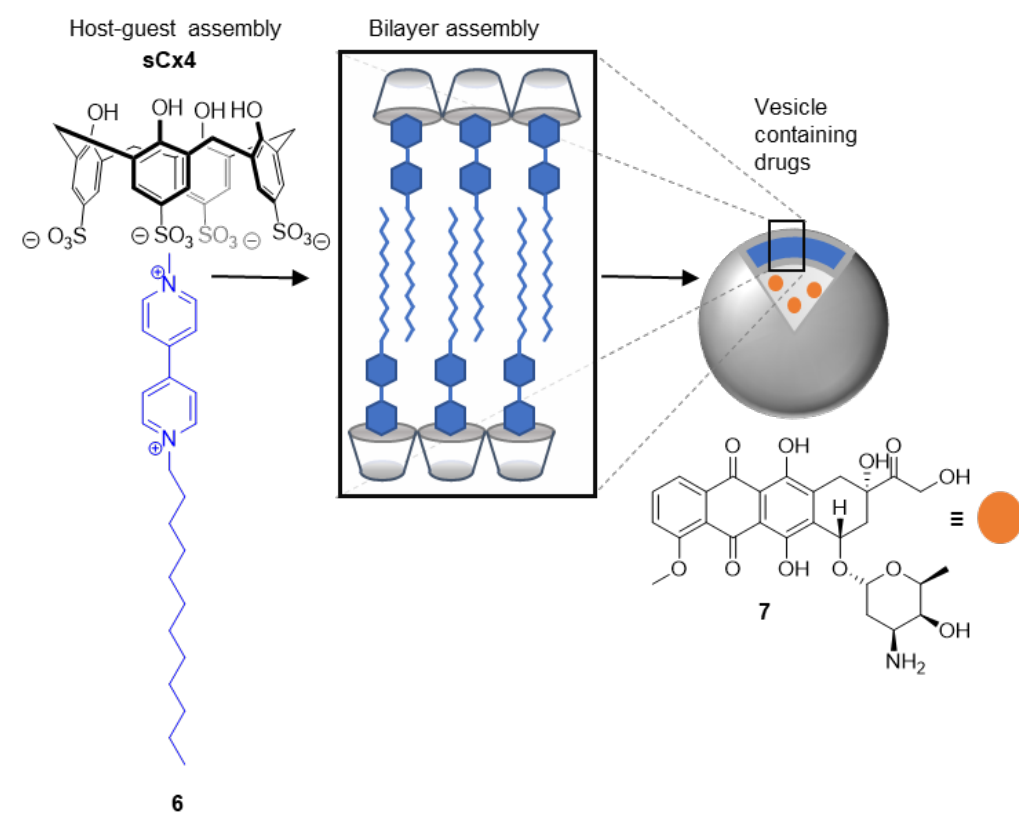

Figure 6. Calixarene-induced aggregation (CIA) has been used to make drug delivery systems.

\section{The challenges of amphiphilic aggregation and charge neutralization-induced precipitation.}

Appending charged functional groups onto an organic host improves water solubility, but often creates an amphiphilic species that is prone to aggregation. At certain concentrations in water, the hydrophobic regions of the host can aggregate into micelles or other structures, while the charged groups face outwards and maximize their contacts with water. Often the hosts' critical aggregation concentration is determined to ensure that host-guest binding studies are conducted under conditions where the host monomer is dominant, instead of studying guest binding by an illdefined aggregate. However, even at low host concentrations the addition of the guest itself can cause aggregation and precipitation. In one set of examples, it has been observed that guests like fluorescent dyes, drugs, surfactants, or proteins form large assemblies with sulfonated calixarenes. This phenomenon is so general that it was given a name: "calixarene-induced aggregation" (CIA). ${ }^{9}$ In one series of studies, this potential nuisance was transformed into a strategy to form stimuliresponsive drug delivery vesicles, as well as other novel materials. For example, the sulfonated calixarene sCx4 formed a host-guest complex with an amphiphilic guest, 6, and together they further assembled into a bilayer vesicle that encapsulated the drug doxorubicin, 7 (Figure 6). ${ }^{10}$

In a related set of challenges, precipitation can occur when charged solubilizing groups encounter certain oppositely charged binding partners that render them effectively neutral and 
decrease solubility. For example, tetra-anionic (8) and tetra-cationic (9) calixarenes form dimeric capsules with each other in polar organic solvents, driven by complementarity of electrostatic charges (Figure 7).${ }^{11}$ Yet, this perfect charge complementarity causes the complex to become water-insoluble and precipitate from aqueous solution. This can be overcome modifying each member of the capsule with different solubilizing groups. For example, by changing the upper-rim sulfonates to carboxylates (10) while also replacing the lower rim alkyl groups for glycol-type groups (11) the capsule forms and remains soluble in water. ${ }^{12}$ In our own group, we have synthesized unpublished calixarenes that contain a symmetric arrangement of two anionic and two cationic functional groups, and we noticed this internal, symmetric charge neutralization routinely renders the molecules almost perfectly insoluble in water (or any other solvent). We have also heard many tales at conferences of work in which the study of a host-guest system in water was impeded because of charge-neutralization-induced precipitation. This work is well known by many, but is almost always unpublished, because of the significant barriers to characterizing precipitating systems and to publishing negative results. The presence of counter-ions from the host, buffer or salt from biological solutions can also changes the solubility of hosts in water. This effect will be explored more in depth in a later section.

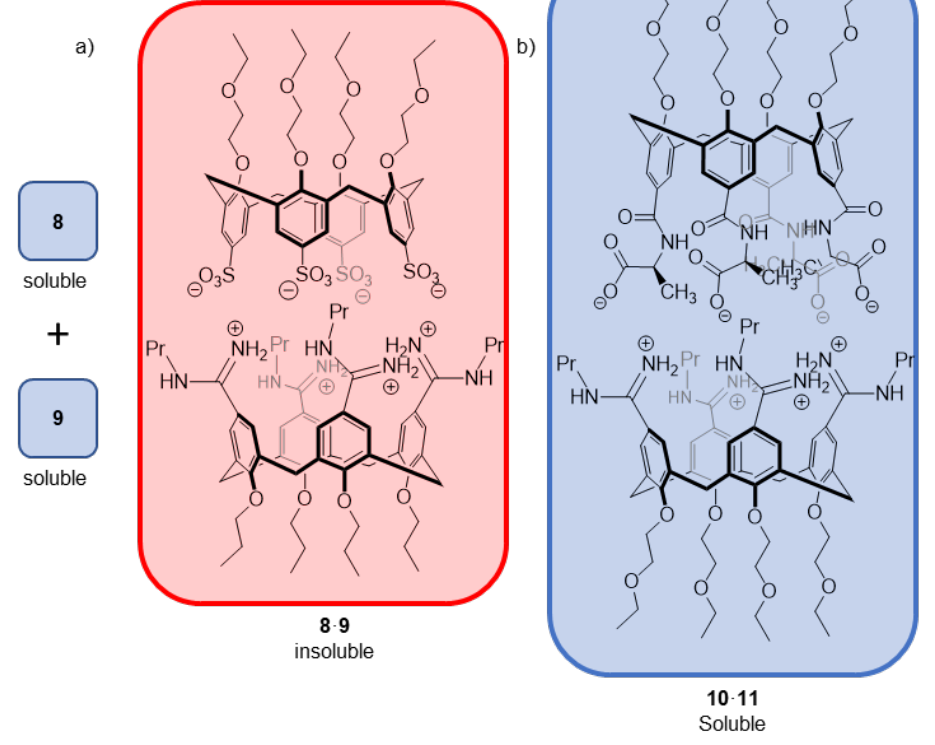

Figure 7 a) Oppositely charged solubilizing groups on $\mathbf{8}$ and $\mathbf{9}$ neutralize each other, rendering the assembly insoluble in water. b) Changing the sulfonates for carboxylates (10) and propyl chains for glycol-type groups (11) increases the solubility of the capsular assembly. 


\section{Host topology, shape, hydration and flexibility}

We often look to Nature for guidance as to which interactions are more prevalent and perhaps most important when forming large assemblies in water. For example, we learn early that hydrogen bonds are responsible for nucleotide base pairing in DNA. However, when hydrogen bonds alone stitch a host and a guest together, assemblies are very sensitive to competition by water. (The assembly of DNA in fact benefits from a large hydrophobic driving force that is often omitted from our earliest lessons on the subject.) Hydrogen bonds alone are rarely enough to bring host and guest together in pure water.

One exception to this rule about hydrogen bonds helps us to understand the influence of host topology on guest binding. Triazole-based hosts can bind $\mathrm{Cl}^{-}$through hydrogen bonds donated by strongly polarized $\mathrm{CH}$ bonds. An acyclic host (12, Figure 8a) with four triazoles binds very weakly in $\mathrm{CD}_{2} \mathrm{Cl}_{2}\left(\mathrm{~K}_{\mathrm{d}} \sim 12 \mathrm{mM}\right)$ and not at all in water. ${ }^{13} \mathrm{~A}$ macrocyclic analog (13, Figure $\left.8 \mathrm{~b}\right)$ binds much more strongly in $\mathrm{CD}_{2} \mathrm{Cl}_{2}\left(\mathrm{~K}_{\mathrm{d}}=125 \mathrm{nM}\right)$, showing how macrocycles (like all hosts in this review) provide an advantage based on preorganized binding surfaces that surround a guest in two dimensions. ${ }^{14}$ But the cryptand-like cage, 14, in Figure 8c contains $9 \mathrm{C}-\mathrm{H}$ derived hydrogen bonds that surround the $\mathrm{Cl}^{-}$in three dimensions. It binds incredibly strongly using only hydrogen bonds $\left(\mathrm{K}_{\mathrm{d}}=10 \mathrm{aM}\right.$ in wet $\left.\mathrm{CD}_{2} \mathrm{Cl}_{2}\right)$ and successfully binds the anion in water and transfers it into dichloromethane. ${ }^{14}$

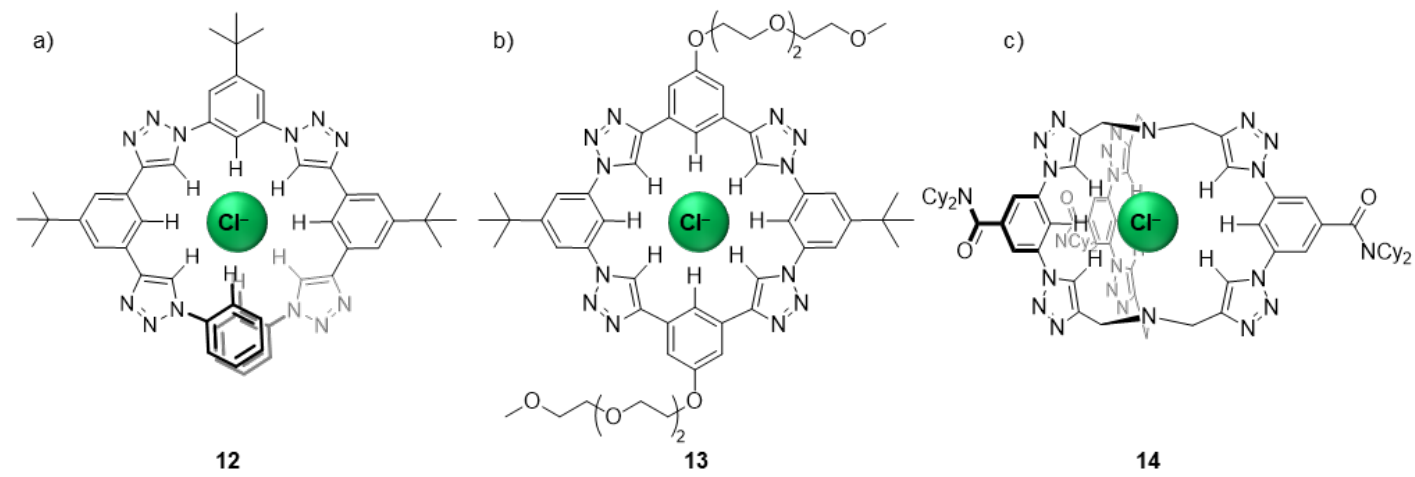

Figure 8 a) A flexible, foldamer-type host (12) binds weekly to chloride $\left(\mathrm{K}_{\mathrm{d}}=12 \mathrm{mM}\right)$ while binding is significantly improved with $b)$ the planar analog $\left(K_{d}=125 \mathrm{nM}\right.$ in wet dichloromethane) and the $\mathrm{c})$ cryptand-like cage $\left(\mathrm{K}_{\mathrm{d}}=10 \mathrm{aM}\right.$ in wet dichloromethane)

In pure water, a host topology that combines a hydrophobic binding motif with adjacent polar interactions is often advantageous. The $\mathrm{CBs}$ have rigid hydrophobic cavities, and 
electronegative ureido carbonyl groups pointing inwards at each portal to stabilize polar or positively charged groups. CB7 binds to neutral guest diamantane (15) strongly in water $\left(\mathrm{K}_{\mathrm{d}}=250\right.$ $\mathrm{pM})$ but binds to the di-cationic analog, 16, 25-fold more strongly $\left(\mathrm{K}_{\mathrm{d}}=10 \mathrm{pM}\right)$ (Figure 9). ${ }^{15}$ The ion-dipole interactions between the two ammonium groups and the carbonyls would be impotent in water if presented on their own, but constitute an effective motif for molecular recognition in water when preorganized, surrounding the guest, and adjacent to host and guest hydrophobic elements.
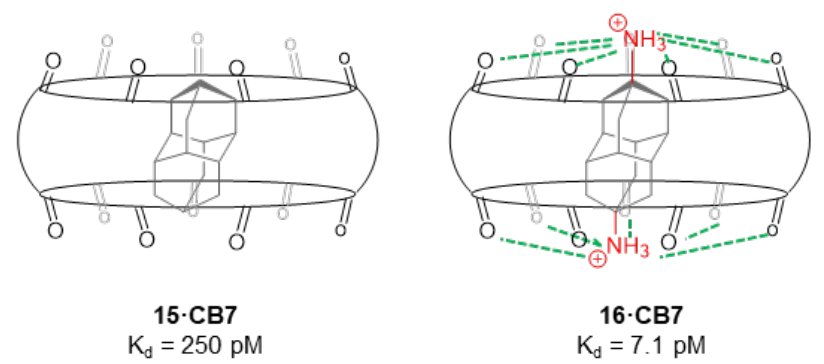

Figure 9. Weak, polar interactions can function in water when adjacent to hydrophobic surfaces. In this example, the ion-dipole interactions provided by cucurbituril carbonyl portals form a much more stable complex with the dicationic guest (16) than with an analogous neutral guest (15). 
a)

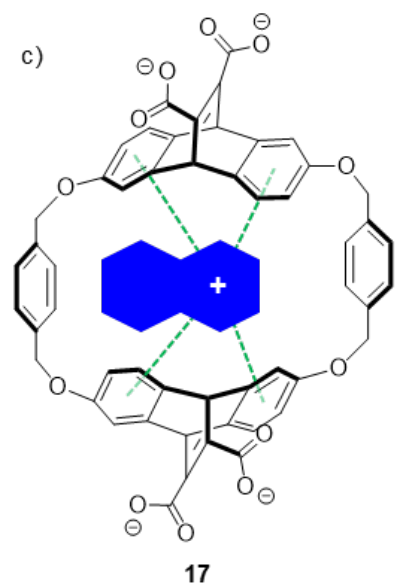

b)
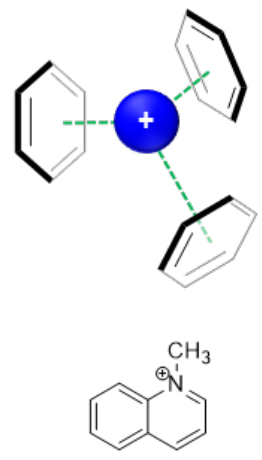

18

$\mathrm{K}_{\mathrm{d}}=0.6 \mu \mathrm{M}$

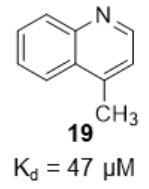

hydrophobic cations

hydrophilic cations

d)

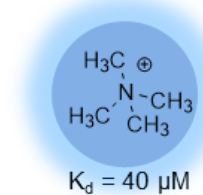

f)

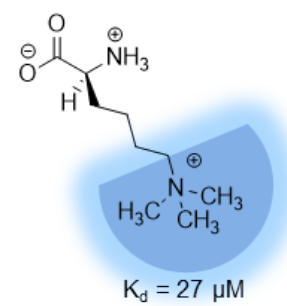

e)

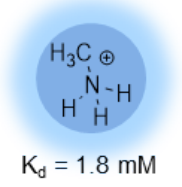

g)

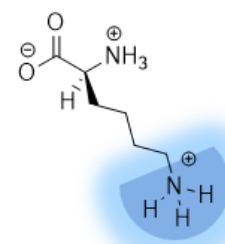

$\mathrm{K}_{\mathrm{d}}=1.9 \mathrm{mM}$

Figure 10. Aromatic faces provide an electron rich surface to interact strongly with cationic guests. a) A simple benzene ring participates in cation- $\pi$ interactions which can be effective for guest binding when $b$ ) the aromatic rings are arranged in a cyclic array. c) Cyclophane $\mathbf{1 7}$ binds preferably to 18 over the isosteric, non-cationic version 19. Host $\mathbf{s C x} 4$ binds to a series of

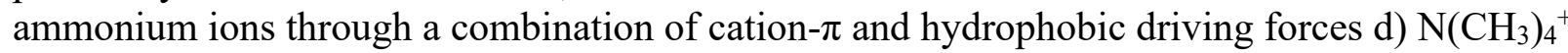
and f) trimethyllysine bind more strongly than their more hydrophilic counterparts e) $\mathrm{CH}_{3} \mathrm{NH}_{3}{ }^{+}$ and g) lysine.

Cation- $\pi$ interactions readily combine both polar and hydrophobic aspects, and as such are especially important in aqueous host-guest chemistry. Aromatic rings provide an electron-rich surface for strong non-covalent interactions with cations (Figure 10a). This interaction is amplified with cyclophane hosts, as there are multiple aromatic rings that create a pocket that is simultaneously hydrophobic (lined by aromatic rings) and electron-rich (lined by the $\pi$ clouds of 
those rings) (Figure 10b). ${ }^{16}$ This inherently provides these hosts selectivity for alkyl ammonium ion guests, which are themselves both cationic and hydrophobic. The combined influence of cation- $\pi$ interactions and hydrophobicity is supported by a few examples involving the comparison of related guests. Cyclophane 17 binds cationic $N$-methylquinolinium, (18) nearly 80 -fold stronger than its neutral, isosteric counterpart, 4-methylquinoline (19), showing that the cation- $\pi$

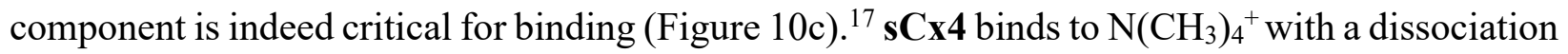
constant of $40 \mu \mathrm{M}$ (Figure 10d) while the more hydrophilic guest, $\mathrm{CH}_{3} \mathrm{NH}_{3}{ }^{+}$, binds much more weakly $\left(\mathrm{K}_{\mathrm{d}}=1.8 \mathrm{mM}\right)$ (Figure 10e), showing that hydrophobicity also plays an important role. ${ }^{18}$ This trend extends to biologically relevant cations, many of which are quite hydrophobic (including many drugs, neurotransmitters, natural products, and certain amino acids and peptides). sCx4 is 70-times selective for a greasy cation, trimethyllysine (Figure 10f) over the un-methylated counterpart, lysine (Figure 10g). ${ }^{19}$ These trends in binding are commonly observed, and can't be explained by the cation- $\pi$ interaction alone; the strength of cation- $\pi$ interactions decrease as the ionic radius of a guest increases, and should actually favour the smaller cations in the absence of any hydrophobic contribution.

The hydrophobic effect alone is also strongly dependent on host topology. An advanced, theoretical understanding of the hydrophobic effect can be found elsewhere. ${ }^{20}$ Here, we will discuss the practical (and sometimes unexpected) consequences of water existing inside host cavities of different shapes and sizes.

Because water makes many strong contacts with itself, as well as charged and polar motifs, it is by exclusion from water that neutral and non-polar motifs associate. How water interacts with a guest and host before and after complexation is a major factor in determining how strong the association will be. The phase separation of oil and water is entropically driven, which leads to the first explanations of the hydrophobic effect that one is typically taught. To explain the observed entropic driving force, it is normally considered that the layer of water solvating hydrophobic surfaces has fewer degrees of freedom in comparison to bulk water. The binding of one hydrophobic surface to another reduces the total surface area that is solvated by water, which liberates some water back into the bulk and increases entropy. This model is sometimes called the 'classical hydrophobic effect' (Figure 11a). 

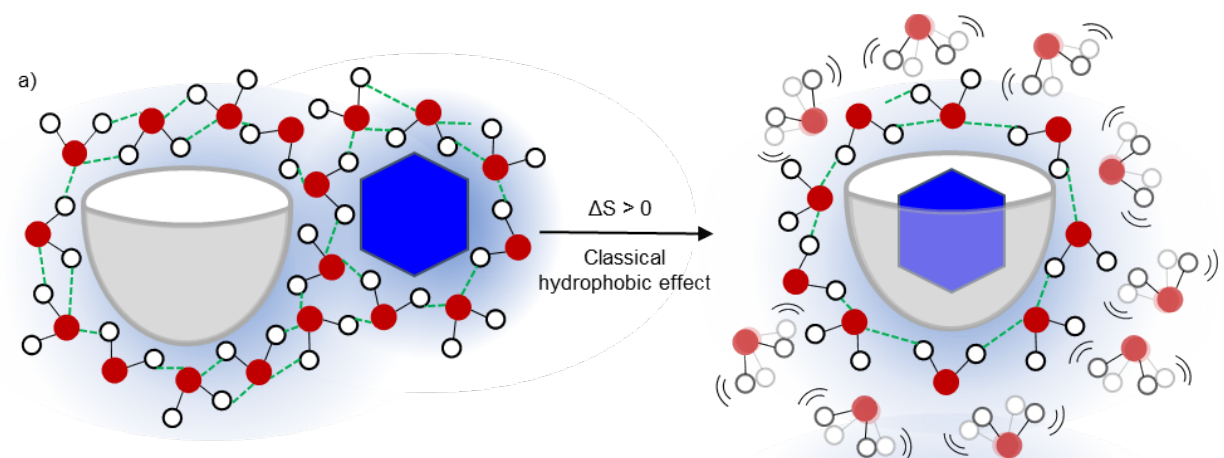

b)
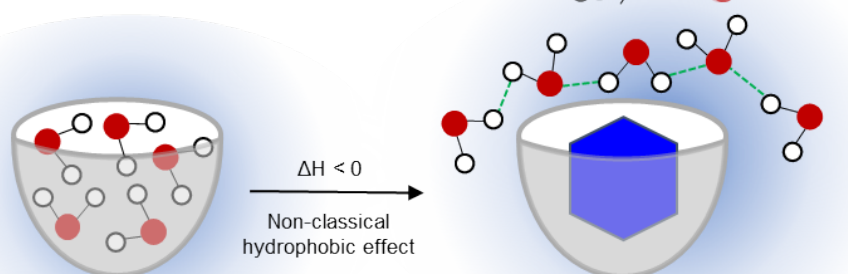

Figure 11. Two simple models that show how entropic and enthalpic driving forces for hydrophobic binding apply in the context of host-guest chemistry. a) Cartoon illustration of the entropy-driven classical hydrophobic effect between a hydrophobic host and its guest. The release of highly ordered water molecules increases their entropy, which drives complexation. b) Cartoon illustration of the enthalpy-driven non-classical hydrophobic effect where it arises for small host cavities. Water encapsulated in small cavities is constrained such that it forms fewer stabilizing water-water hydrogen bonds than it can in bulk solvent. Release of these water molecules upon guest binding leads to a net increase in hydrogen bonds and a favourable decrease in enthalpy.

Defying the expectations of this simple model, host-guest binding in water is often also accompanied by a favourable change in enthalpy. The model that explains these observations, called the 'non-classical hydrophobic effect,' centers on the unusual hydration of small, rigid cavities. Small hosts and other highly concave surfaces put unusual constraints on their solvating waters. They can fit, but they can't form their normal complement of hydrogen bonds with neighboring waters. The few encapsulated water molecules can no longer make the same number of hydrogen bonds as they would in the bulk. This is considered to be a pool of "high-energy water molecules" (although the term is controversial). Guest binding leads to the return of this water back into bulk where more water-water hydrogen bonds can form, giving a structural explanation for the origin of the observed favorable enthalpies of binding (Figure 11b). If the host's volume becomes so small that not even a single water molecule can be encapsulated, the host is considered prematurely "de-solvated" from water and welcomes a suitably sized guest. It has been proven 
both experimentally and computationally that CB5 — the smallest member of the cucurbituril family - is too small to encapsulate any water molecules and that only a vacuum exists within the cavity (Figure 12). ${ }^{21}$ This drives the encapsulation of noble gases in water. Interestingly, hosts like $\mathbf{s C x} 4$ and $\boldsymbol{\alpha}-\mathbf{C D}$ can't bind noble gases, in spite of their cavity size and chemical functionality being generally similar to $\mathbf{C B 5}$. Both $\mathbf{s C x} 4$ and $\boldsymbol{\alpha}-\mathbf{C D}$ cavities have conical shapes that allow at least partial access to solvating waters, giving a concrete example of how more 'closed' topology hosts can have better guest binding than those that are more 'open' to solvation.

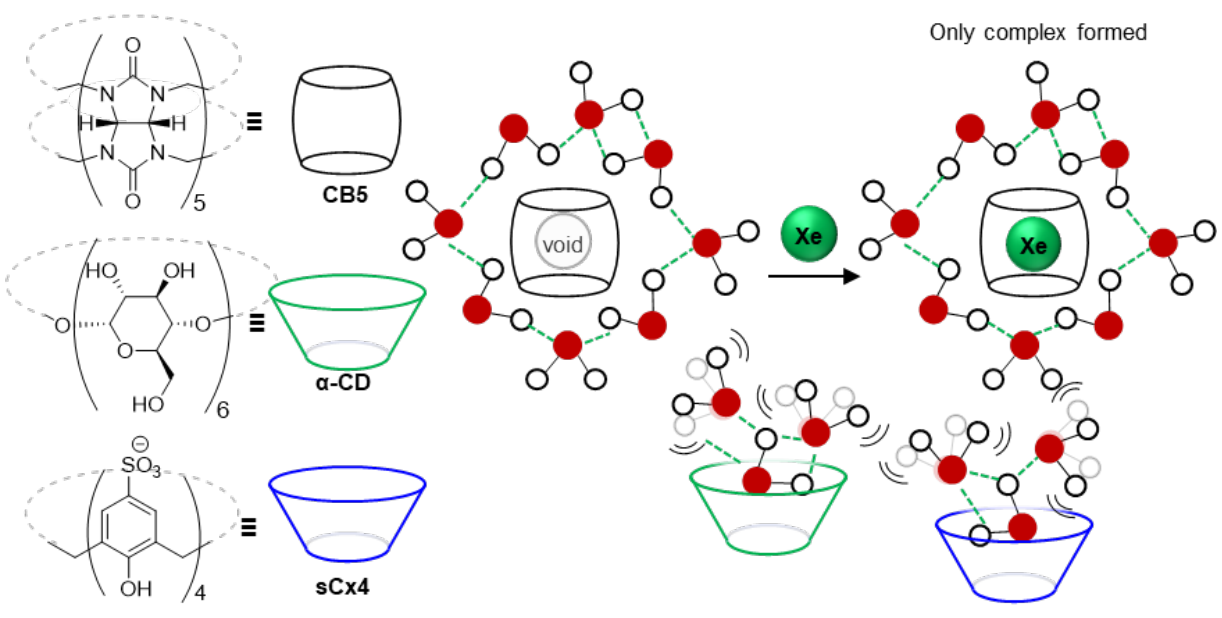

Figure 12. The interplay of host topology and solvating waters can influence guest binding. CB5 is the only host from among three similarly sized hosts that sequesters Xenon from water. CB5 contains an empty de-solvated cavity that is filled perfectly when noble gases complex. $\boldsymbol{\alpha}-\mathbf{C D}$ and $\mathbf{s C x} 4$ have similar sizes but different topologies, and make favourable interactions with water while not binding Xenon.

If it fits, it sits. Complementary host-guest shape aids in the formation of strong complexes. While essentially every host-guest complex holds lessons in shape complementarity, we choose a pair of cyclophanes, $\mathbf{2 0}$ and $\mathbf{2 1}$, to teach this lesson. The two hosts are very similar in chemical functionality, but vary in cavity shape in a way that favors the binding of different, closely related guests (Figure 13). ${ }^{22}$ Host 20 contains a single carboxyphenyl linker, creates an overall more rounded binding pocket, and selectively binds to trimethyllysine (Kme3), an amino acid with a cationic element that has a complementary, rounded shape. Host 21 contains a carboxynaphthyl linker, which creates a more rectangular binding pocket, and instead selectively binds to asymmetric dimethylarginine (aRme2). aRme 2 is an amino acid with a flatter and more rectangular 
cationic side chain. The subtle change in cavity shape increases the selectivity by $\sim 2$-fold for aRme2 over Kme3.
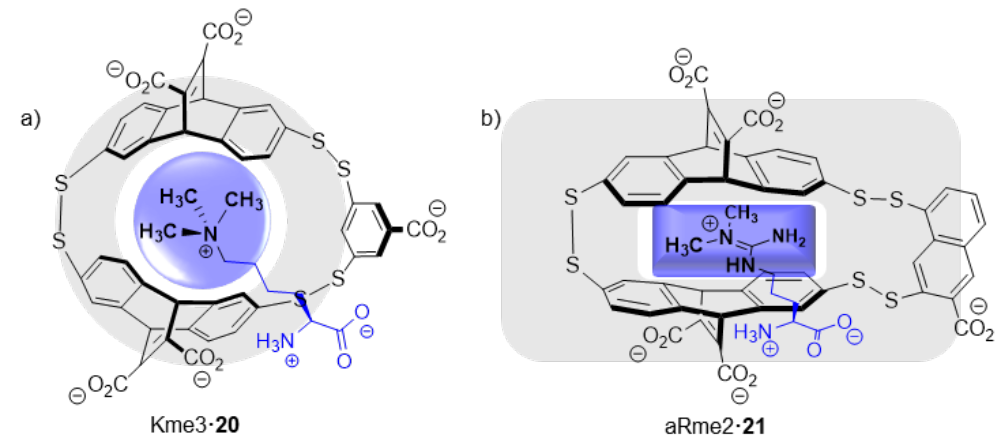

Figure 13. Different cavity shapes influence guest binding. a) $\mathbf{2 0}$ has a spherical cavity that complements the side chain of Kme3 while b) $\mathbf{2 1}$ has a rectangular cavity that is better suited for a planar guest like aRme2.
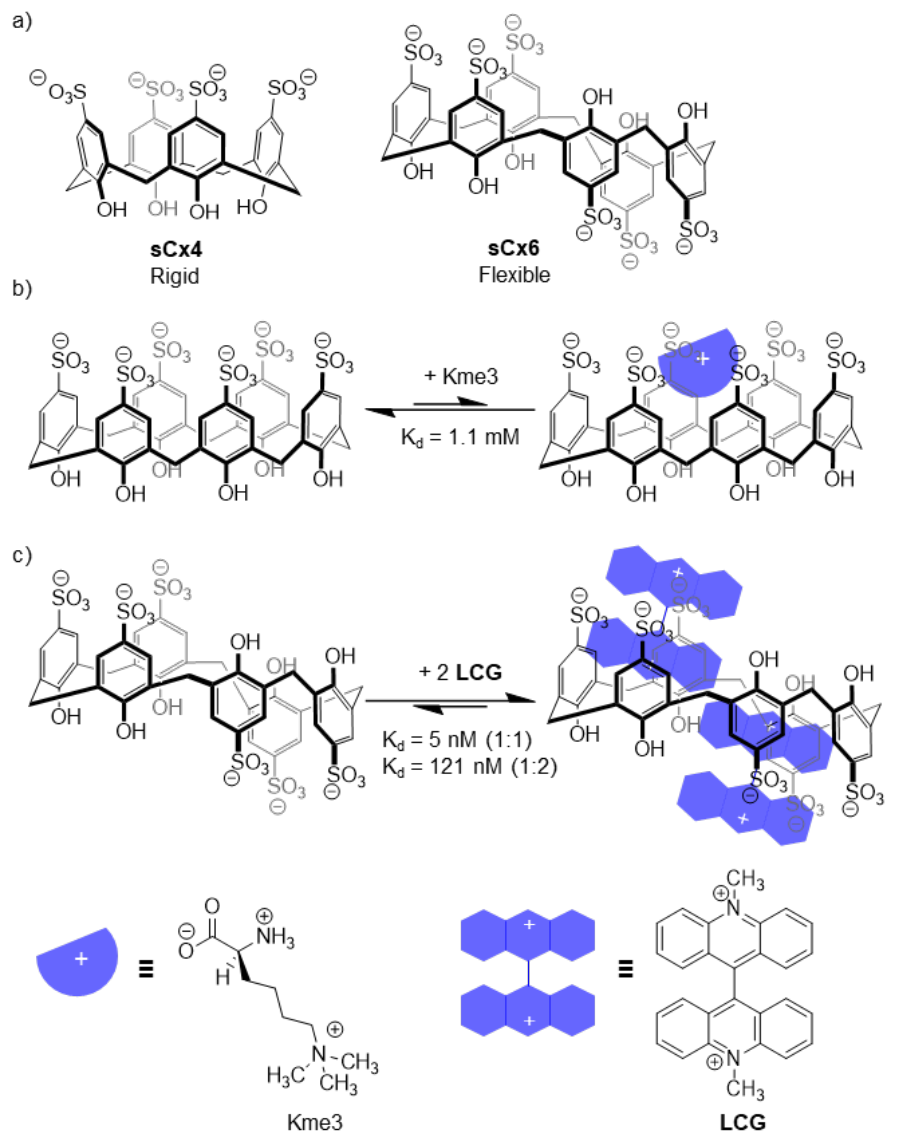

Figure 14. Host flexibility can dramatically alter guest binding in unpredictable ways. a) sCx4 offers a more rigid binding site than sCx6. b) Trimethyllysine (Kme3) binds well to sCx4 (see text), but very poorly to sCx6. c) A larger guest, lucigenin (LCG), is binds very strongly to both sCx4 (1:1 complex, not shown), and $\mathbf{s C x 6}$ (both 1:1 and 1:2 complexes). 
Host flexibility is a distinct aspect of shape and topology that must be understood in order to create strong host-guest complexes.

As mentioned earlier, creating preorganized, rigid hosts is favourable for guest binding, and also helps to encode guest selectivity. $\mathbf{s} \mathbf{C x} 4$ provide a rigid, well-defined pocket that binds strongly to organic cations such as trimethyllysine $\left(K_{d}=28 \mu \mathrm{M}\right)$ and lucigenin, LCG $\left(K_{d}=35\right.$ $\mathrm{nM}) .{ }^{19,23}$ Larger, more flexible analogs of $\mathbf{s C x} 4$ are available in the forms of the 6-membered calixarene, sCx6 (Figure 14a). This ultimately changes the binding topology and stoichiometry which is reflected in the binding strengths to the same guests. $\mathbf{s C x 6}$ easily interconverts to adopt many conformations. It does not bind strongly with trimethyllysine $\left(K_{d}=1.1 \mathrm{mM}\right)$, in spite of having more hydrophobic surface area and more negative charge than $\mathbf{s C x} 4$ (Figure 14b). ${ }^{19}$ Yet sCx6 has an affinity for the larger guest lucigenin, LCG $\left(\mathrm{K}_{\mathrm{d}}=5 \mathrm{nM}, 1: 1\right)$, which is similar to that determined for the $\mathbf{s C x}$ 4-lucigenin complex, and also displays the ability to form 1:2 complexes with this guest $\left(\mathrm{K}_{\mathrm{d}}=122 \mathrm{nM}\right.$, Figure $\left.14 \mathrm{c}\right) .{ }^{24}$ These examples show that it is difficult, and often inadvisable, to predict the behaviours of highly flexible hosts.

a)
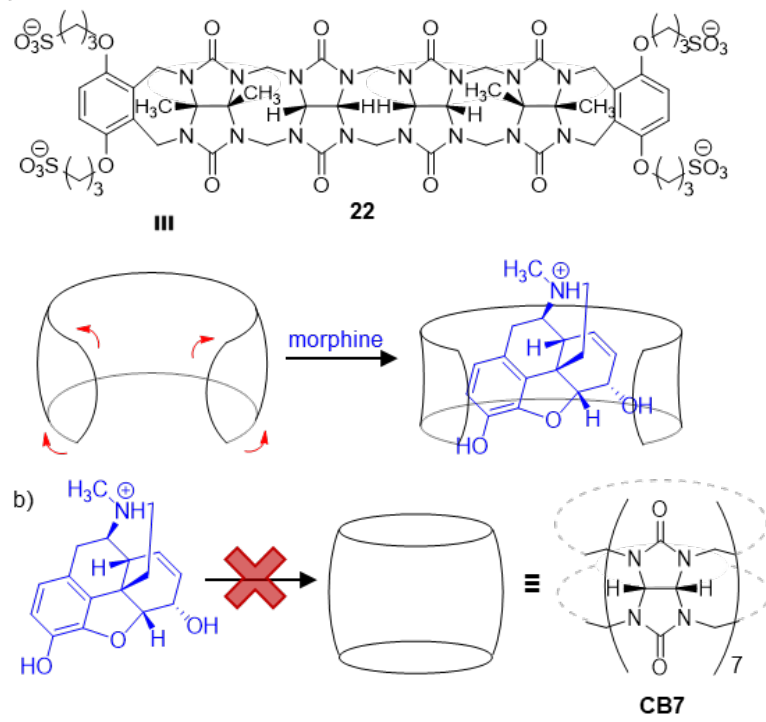

Figure 15. Host flexibility facilitates an induced fit for larger guests. a) Acyclic cucurbituril, 22, can flex to bind with large guests like morphine whereas b) cyclic cucurbiturils are inflexible and bind poorly to morphine. 
There are some scenarios in which giving hosts small degrees of added flexibility provides a clear advantage. If a rigid host is only a fraction of an Angstrom too small, it will absolutely refuse to bind a guest. This is due to the incredibly high penalties for van der Waals interactions at distances that are too short (i.e. steric overlap). For example, CB7 binds many ammonium ion guests very strongly but does not bind morphine at all. Compound 22, which is an acyclic, Ushaped $\mathbf{C B}$ analog binds strongly to morphine $\left(\mathrm{K}_{\mathrm{d}}=2 \mu \mathrm{M}\right)$ and also binds a diverse suite of similar, large guests (Figure 15). ${ }^{25}$ The acyclic, cleft-like host is rigid enough to encode strong binding, but has a slight flexibility that allows it to accommodate guests that can't be retained by its cyclic counterpart. On the extreme, however, it is well known that the binding pocket of a completely flexible host will undergo a hydrophobic collapse upon itself when dissolved in water, which completely turns off guest binding. These examples show how a balance of rigidity and flexibility can be advantageous in creating aqueous host systems.

\section{Salts perturb host-guest systems in aqueous solution}

Salts are ubiquitous in nature, and often negatively impact host-guest systems. In order to design hosts that will function in a biological medium, the system is typically first studied in buffered water to mimic the $\mathrm{pH}$ levels (and sometimes ionic strength) of the natural solution. The type of salt and the choice of buffer used can drastically change how the host behaves.

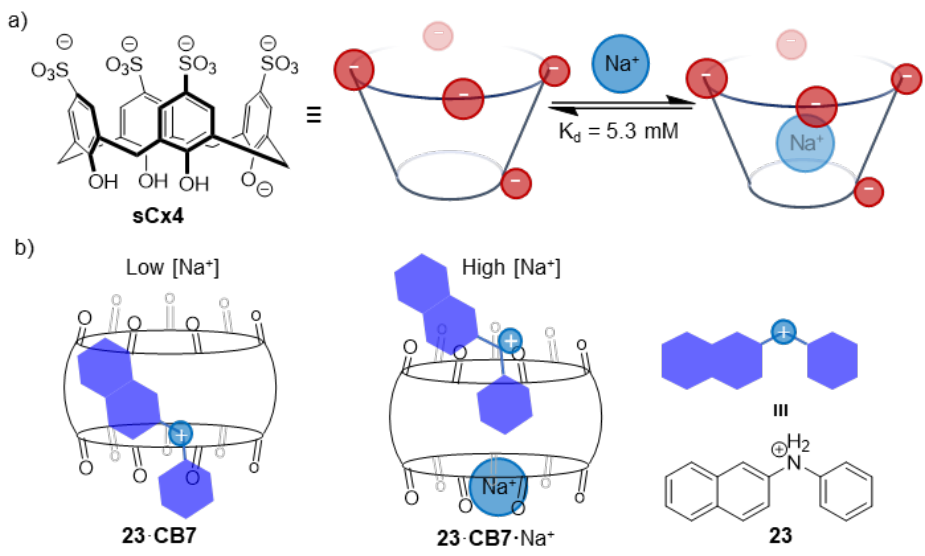

Figure 16. Direct influences of counterions over host-guest binding. a) Buffer-derived $\mathrm{Na}^{+}$from buffers are guests for $\mathbf{s} \mathbf{C x}$, and can disrupt the binding of intended guests. b) $\mathrm{Na}^{+}$binds with the carbonyl portals of cucurbiturils and modulates guest binding and orientation. $\mathrm{Na}^{+}$can acts as a competitor, favouring the dissociation of the naphthyl motif in $\mathbf{2 3}$, or act like a cap which favours the association of the same guest via its phenyl group. 
Hard metal cations can act as competitive guests for polar sites in supramolecular cavities. The counter-cation in most anionic buffers (phosphate, citrate, etc) is often overlooked, but in fact can bind directly to hosts that have anionic solubilizing groups or other electron-rich motifs. $\mathbf{s C x 4}$ is best known to bind with cationic ammonium guests, but also binds $\mathrm{Na}^{+}$with $\mathrm{K}_{\mathrm{d}}=5.3 \mathrm{mM}^{26}$ Although this is a weaker binding event than with other ammonium guests, if one considers that the buffer concentration is often as high as $150 \mathrm{mM}$ (see below), then nearly every host can be occupied by $\mathrm{Na}^{+}$, (Figure 16a). The addition of salt or acid $\left(\mathrm{H}_{3} \mathrm{O}^{+}\right)$helps increase the solubility of cucurbiturils in water because the cation binds to the electronegative ureido carbonyls. Extensive mechanistic studies have shown that $\mathrm{Na}^{+}$and other simple cations can bind to both carbonyl portals and modulate guest encapsulation. This either weakens the complex (when the cation acts as a competitive guest), or increases the strength of host-guest complex (when the cation directly enhances binding). For example, CB7 binds with a ditopic guest, 23, via the phenyl or naphthyl ring but prefers the latter at low concentrations of $\mathrm{Na}^{+} \cdot{ }^{27}$ At high concentrations of $\mathrm{Na}^{+}$, the cation acts as a competitor and weakens the naphthyl inclusion, flipping the host's preferences by acting as a stabilizing cap for the phenyl inclusion complex (Figure 16b).

To avoid unfavourable interactions of salt with various hosts, a low buffer concentration is ideal. If must be noted that buffers should always be at least 10-fold more concentrated than the ionizable species they are buffering, in order to ensure sufficient buffering capacity to maintain a constant $\mathrm{pH}$ value. However, when translating these systems to real biological solutions, it is hard to escape the 100's of mM concentrations of various ions that exist in Nature.

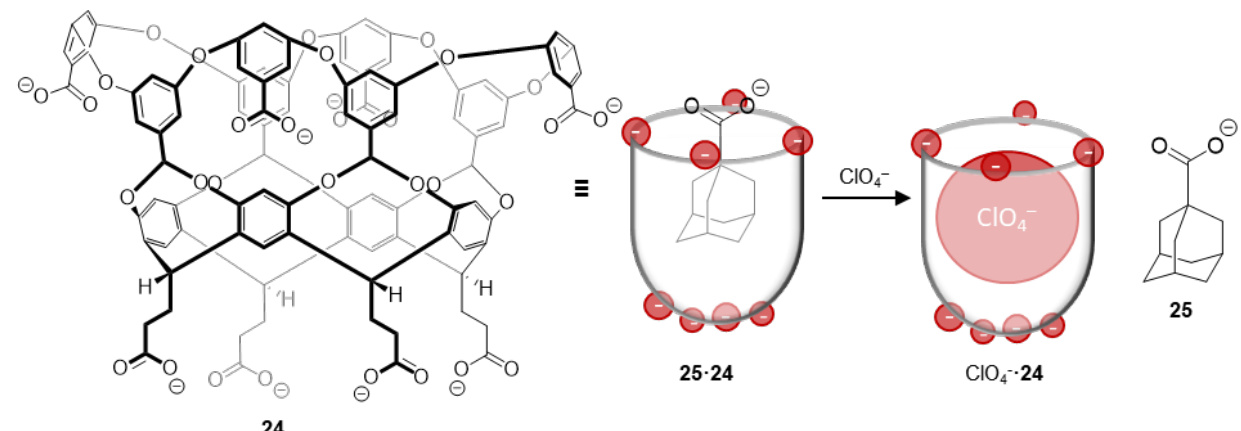

Figure 17 The association between $\mathbf{2 4}$ and $\mathbf{2 5}$ is perturbed by chaotropes like $\mathrm{ClO}_{4}^{-}$as it directly competes for the hydrophobic binding pocket.

Soft, polarizable ions can act as direct competitors for hydrophobic cavities. Anions such as $\mathrm{I}^{-}, \mathrm{ClO}_{4}^{-}$, and $\mathrm{SCN}^{-}$are polarizable and are called chaotropes, due to historical association with the Hofmeister series of ions. Chaotropic ions associate with hydrophobic surfaces, even 
outcompeting guests for the binding pocket. For example, the host-guest complex of $\mathbf{2 4}$ and $\mathbf{2 5}$ weakens in the presence of increasing concentrations of $\mathrm{NaI}, \mathrm{NaClO}_{4}$, and $\mathrm{NaSCN}$ (Figure 17) NMR studies directly show that perchlorate is encapsulated within the $\mathbf{2 4}$, preventing $\mathbf{2 5}$ from binding. ${ }^{28}$ This effect becomes more dramatic when anions increase further in size and polarizability; such anions are referred to as super-chaotropic, and their binding to host pockets can even be used as a design element in its own right. ${ }^{29}$ The chaotropic effect, which explains how such ions bind strongly to their partners, is relatively new yet has already been used to create complex supramolecular structures that function in water and is anticipated to lead to more advances in the near future. ${ }^{29}$

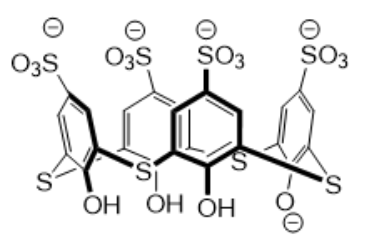

26

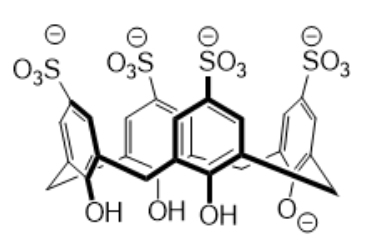

$\mathrm{sCx} 4$

Figure 18 Millimolar concentrations of salt neutralize the charge on highly charged hosts and can render them insoluble in water. 26 and $\mathbf{s C x} 4$ are similar, but $\mathbf{2 6}$ precipitates from solution much more readily upon addition of $\mathrm{NaCl}$.

High concentrations of salt can induce precipitation when ions condense onto charged hosts. Earlier, we described how charged hosts can precipitate upon the association of an oppositely charged guest. Host solubility in water can also be affected by salt when the buffer or solution counterions associate and neutralize the charge on the host (Figure 18). Many examples of such negative results go unreported, but one useful example can teach the lesson. $\mathbf{s C x} 4$ and its corresponding thia-calixarene derivative (26) were used to encapsulate ketone and alcohol guests in water, prior to a 100 -fold excess of $\mathrm{NaCl}$ being added to neutralize the hosts, precipitate the host-guest complexes, and allow their isolation (Figure 18). ${ }^{30}$ The authors reported that there was a clear preference for $\mathbf{2 6}$ to capture guests and salt-out more readily than $\mathbf{s} \mathbf{C x}$, despite both bearing four sulfonates along the upper rim and having very similar structures. This shows that it is not simple to predict how any given class of host will behave in a certain concentration of a salt. When problems are encountered, we recommend changing the identities of buffers and supporting solutes, because subtle changes can solve precipitation problems in unexpected ways. In some cases, the presence of salts can stop short of inducing complete precipitation, and instead induce 
host aggregation or assembly in a way that can be beneficial for their function. Several of the hosts in the next sections are examples of this kind of outcome.

\section{Exemplary systems that operate in the presence of salts and buffers}

An understanding of how hosts behave in salty water aids in the design of supramolecular systems that carry out their functions in a biological context. While there are many examples of hosts that bind with peptides or proteins, that bind to lipids and/or act as bio-active surfactants, and that act as bio-compatible reaction vessels, this discussion will focus on a series of anionic hosts that act as sensors, as well as some key relatives that extend the lesson. Sensors are particularly informative for the current discussion, as the supramolecular systems that target biological analytes need to generate a detectable output in water, while using binding mechanisms that are resistant to salts and other biological solutes.

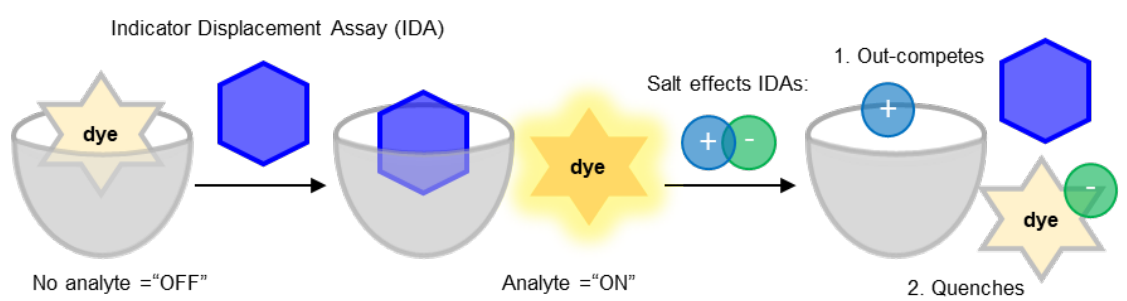

Figure 19. Indicator Displacement Assay (IDA) is used to detect analytes by displacement of a quenched dye. Salt can affect IDAs either acting as a competitor or quenching the dye.

Transforming a host into a chemosensor requires the integration of a reporter motif or molecule that signals when an analyte has been bound by the host. Dyes are common reporters as their optical properties can be sensitive to the environment and to the presence of other molecules. There are various photophysical mechanisms that modulate the response of dyes, and one common strategy is through indicator displacement assays (IDAs), as illustrated in Figure 19. The dye/indicator is encapsulated by the host which creates an "analyte absent" optical state. Once an analyte is present, it displaces the indicator into the bulk water, which induces another optical state of "analyte present". ${ }^{31}$ Some examples of hosts exist in which the hosts have been structurally modified to include this dye molecule tethered covalently to their structure. However, many more supramolecular sensors simply use an added, external dye molecule to avoid the synthetic challenges of creating dye-integrated hosts. Although these sensors are often easily constructed, 
they suffer from the same challenges as any aqueous host-guest system - chemosensors that target biological analytes must work in salty water. Salts can not only act as competitors (see above), but can also directly perturb the photophysical properties of the indicators.

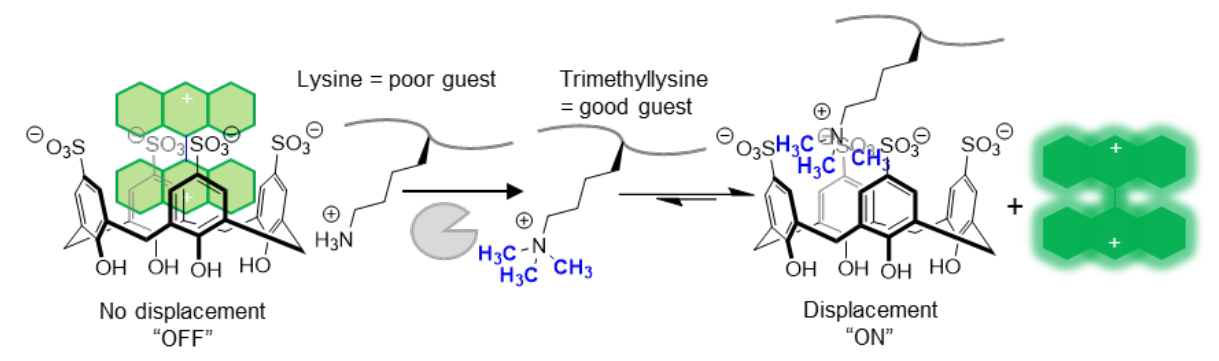

Figure 20. sCx4 and lucigenin (LCG) are used as a conventional IDA sensor to monitor a methyltransferase reaction, giving a turn-on fluorescence response when trimethyllysine is formed by the enzymatic reaction. The use of low $\mathrm{NaCl}$ concentrations minimizes potentially deleterious salt effects.

In spite of these challenges, some examples of supramolecular sensors teach key lessons about compatible buffers and how to use salt effects as an advantage. The two following examples who how the effects of buffer and salt can be considered predictively and/or empirically in order to provide supramolecular chemosensors that can operate in complex aqueous solutions.

In the first example, sCx4 was used to detect the product formation of an enzymatic reaction in real-time when combined with lucigenin (LCG) as the dye component in an indicator displacement assay. ${ }^{32} \mathbf{s C x 4}$ binds to LCG and quenches its emission. One sCx4-LCG assay monitored LCG's fluorescence increase as the dye was displaced from the sCx4 cavity by a trimethyllysine-containing peptide, which was the product of an enzymatic methylation reaction. A glycine buffer (5 mM, pH 10) avoids the presence of large amounts of $\mathrm{Na}^{+}$and/or $\mathrm{Cl}^{-}$that might have been present in other typical buffers. ${ }^{32}$ As discussed previously, $\mathbf{s C x} 4$ binds with $\mathrm{Na}^{+}$directly in a way that can compete with guest recognition. More importantly, $\mathrm{Cl}^{-}$and other halides bind to LCG directly and quench its fluorescence. ${ }^{33}$ 


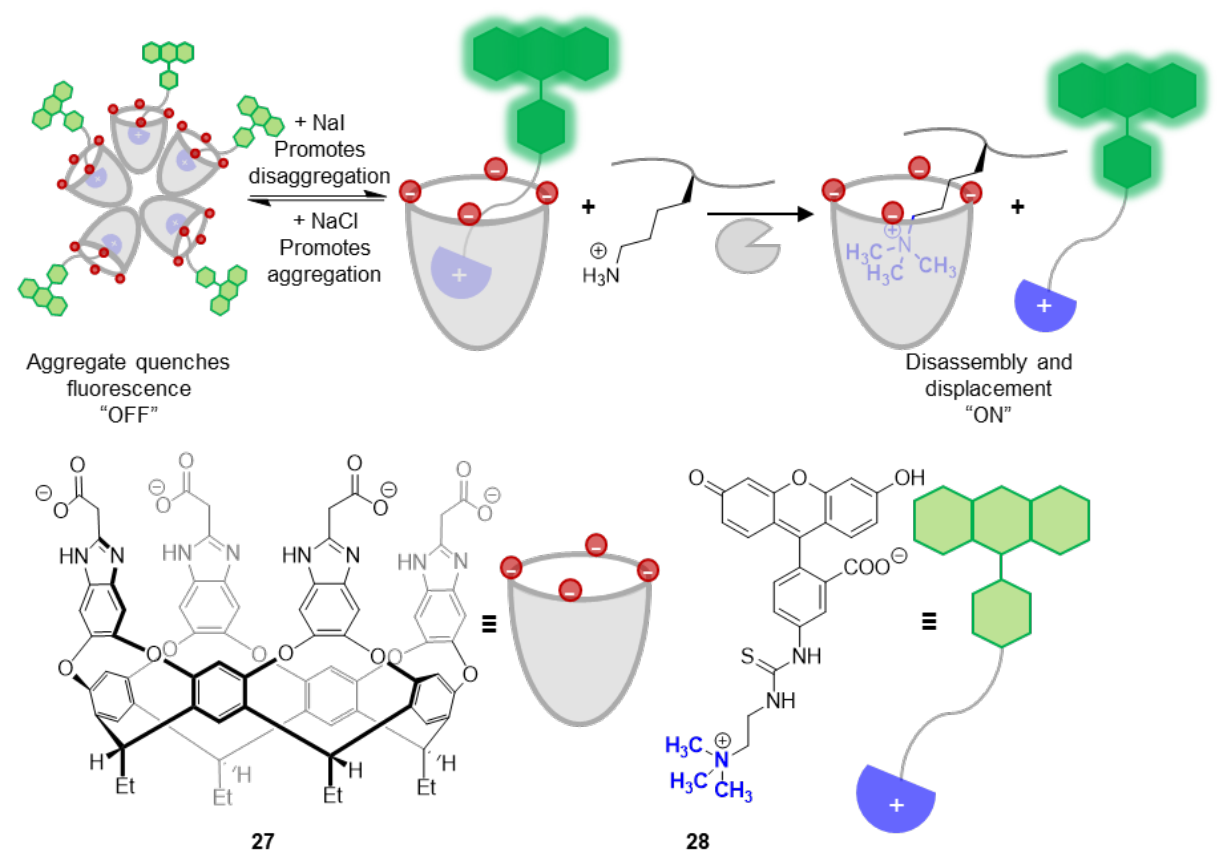

Figure 21. Aggregation-quenched IDA reports on the progress of a demethylase-catalyzed reaction. The sensitivity is influenced by Hofmeister series anions that modulate the formation of the quenched aggregate.

A different example shows how salt effects can provide a functional advantage. In this case a deep-walled cavitand, 27, was paired with a trimethylammonium-fluorescein conjugate, 28, as the displaceable indicator and was used to detect the progress of an enzymatic demethylation reaction (Figure 21). ${ }^{34}$ In this case the IDA works as a turn-off sensor. Host 27 first binds the trimethyllysine-containing substrate peptide, leaving indicator $\mathbf{2 8}$ uncomplexed and emissive. Once the enzyme creates the unmethylated peptide, indicator $\mathbf{2 8}$ associates with the host. However, $\mathbf{2 8}$ does not participate in a typical indicator displacement scheme when encapsulated by $\mathbf{2 7 -}$ instead the amphiphilic dye-host complex forms micelles in which the dye is quenched by aggregation effects. The complex assembly strength was tested in the presence of various salts (e.g. $\mathrm{NaCl}$ and $\mathrm{NaI}$ ). Each salt had a unique influence on the sensor's aggregation, which in turn affected the sensitivity of the assay. 


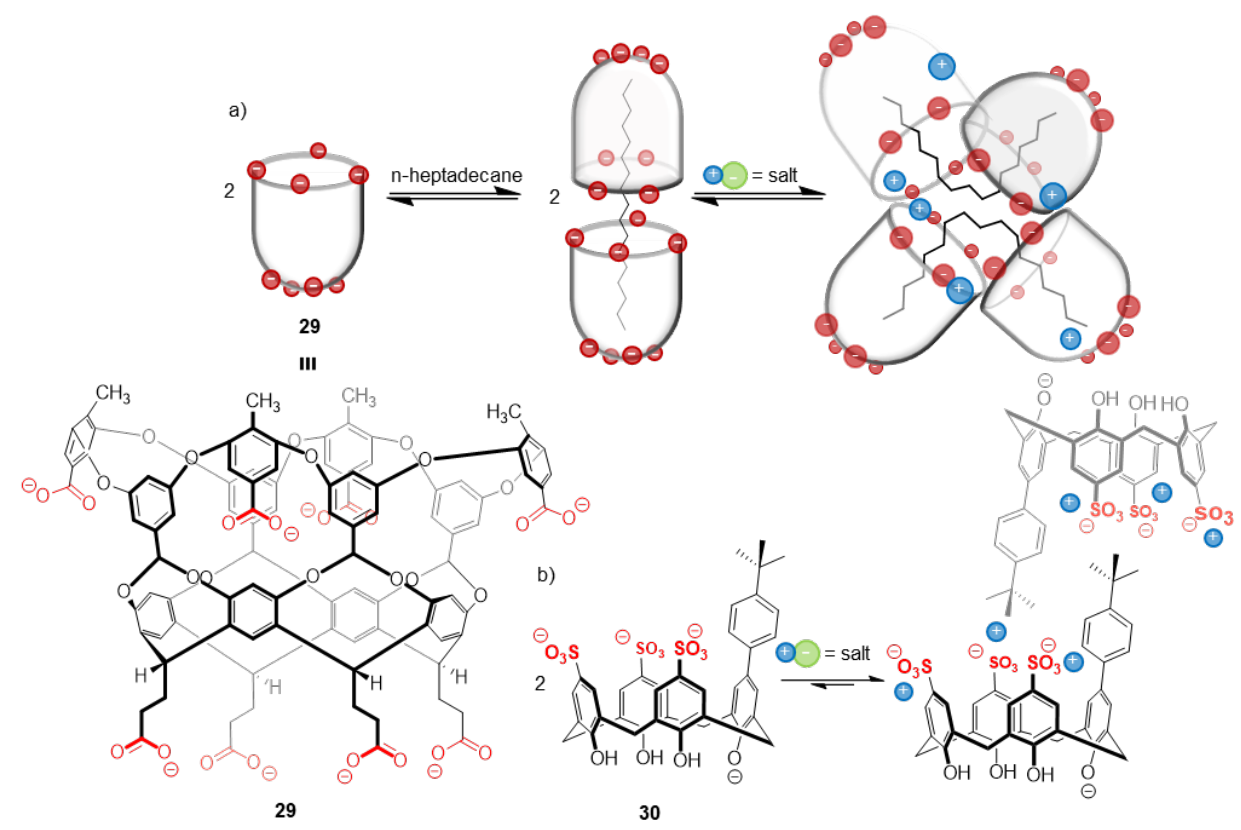

Figure 22. Controlled assembly and salt effects for like-charged host molecules. a) An anionic cavitand (29) forms a dimer with a -16 overall charge to encapsulate n-heptadecane, and upon the addition of salt is induced to form a tetramer with -32 overall charge while enclosing two guests. b) A sulfonated calixarene bearing hydrophobic pendant arms (30) form homodimers with a -8 overall charge in water, and those anionic dimers becomes thermodynamically stronger in presence of added salts.

One final set of examples teaches about a surprising, yet seemingly general motif for salttolerant assemblies. Hosts that combine a significant amount of negative charge along with hydrophobic recognition motifs are often able to assemble with similar partners in salty solutions, and even be promoted by salt. The Gibb cavitand 29 self-assembles into dimers around hydrophobic guests despite forming a complex with overall -16 charge. However, with the addition of various salts, the assembly changes to a tetrameric species (4:2) with an overall -32 charge. The highly charged assembly is presumably stabilized by the binding of cations to anionic groups in order to reduce the overall repulsion among monomers (Figure 22a). ${ }^{35} \mathrm{~A}$ family of calixarenes each functionalized with single, rigid, hydrophobic arms display a related behaviour, forming anionic dimers in aqueous solutions that get stronger upon addition of added salt (Figure 22b). ${ }^{36}$ The operating principle is the same in both cases: hydrophobic aggregation is balanced against the mutual repulsion between like charges in each monomer; salts act to reduce the mutual electrostatic repulsion and strengthen the hydrophobic effect; the strength of each 
assembly is increased in the presence of salt; the overall negative charge is enough to keep the well-ordered assemblies in solution.

\section{Systems that function in real biological media}

The goal for many water-compatible supramolecular systems is to operate in real biological solutions, which can be both complex and unpredictable. Supramolecular hosts offer selective and potent recognition sites that mimic those of biomolecules in Nature. However, an additional challenge for these supramolecular tools is to operate in solutions that are even more complex and less controlled than the salty, buffered aqueous solutions that have been discussed above. Biofluids such as serum, saliva, and urine contain a mixture of salts, proteins, and organic metabolites that vary in concentrations over time and between individuals. ${ }^{37}$ This complexity and heterogeneity is further amplified in cells and whole organisms. Examples of biofluid-compatible chemosensors and medicinally relevant systems that operate in cells and whole organisms are discussed below. It is generally harder to teach general lessons from such diverse material. The purpose of this section is to inspire tutorial readers with examples of the achievements of host-guest chemistry under the most complex and challenging of biological conditions.

\section{Chemosensors that function in biofluids}

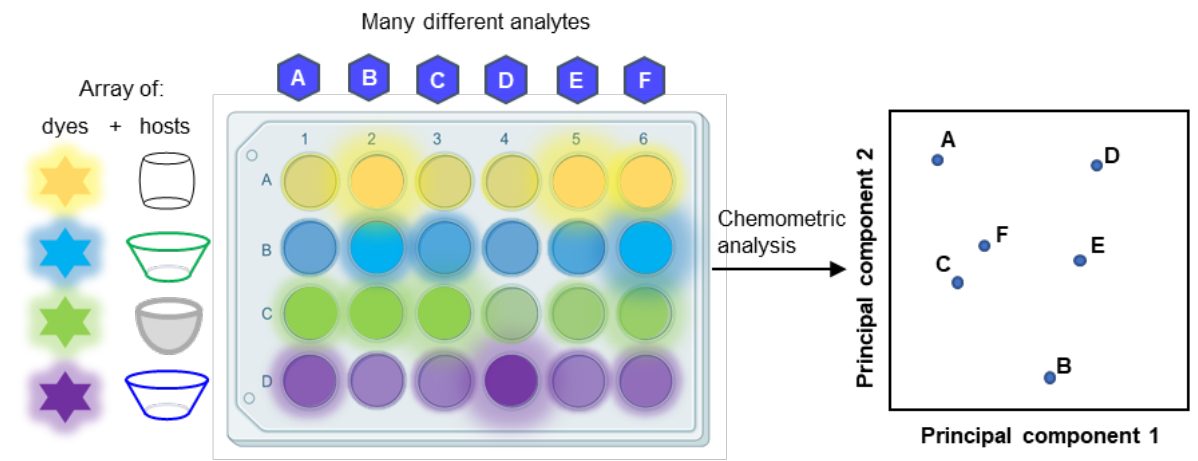

Figure 23. A generic representation of a host-guest based sensor array. An array of dyes paired with promiscuous hosts create IDAs that detect many different analytes, each to a varying extent. This produces a complex 'fingerprint' of output data which is simplified and analysed through chemometric analyses in order to distinguish each analyte. 

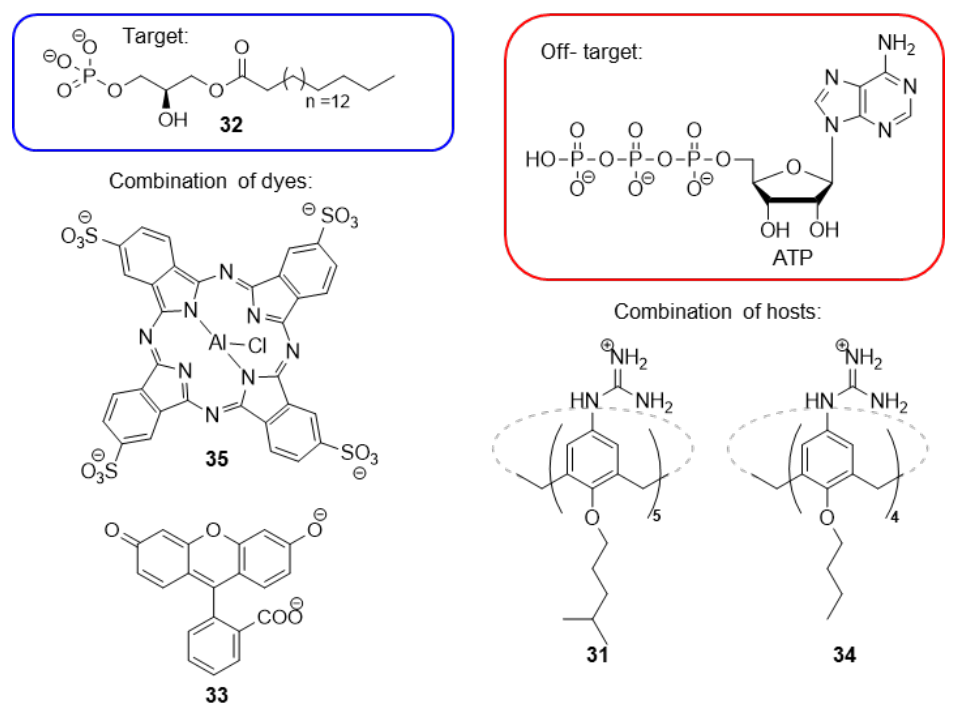

Figure 24. A combination of two different calixarenes $(\mathbf{3 1}, \mathbf{3 4})$ and dyes $(\mathbf{3 3}, \mathbf{3 5})$ detect and discriminate a cancer biomarker, lysophosphatidic acid (32), from an off-target molecule, adenosine triphosphate, ATP. The system functions in plasma.

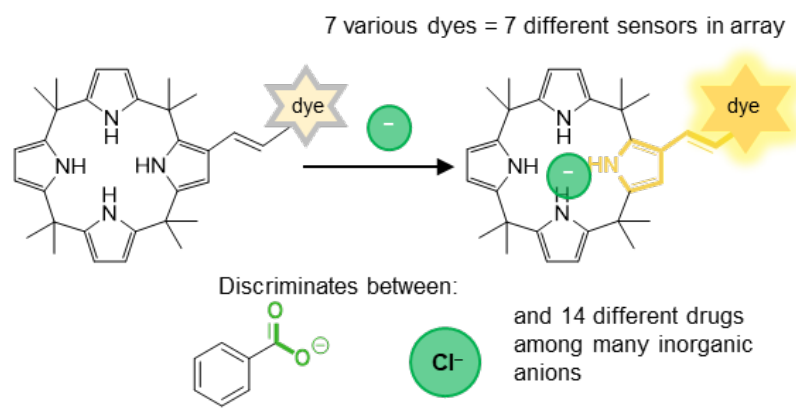

Figure 25. An array of 7 dye-integrated calix[4]pyrrole chemosensors detect over-the-counter carboxylate drugs in urine despite also forming weak interactions with other inorganic anions.

Many chemosensors that function in biofluids operate within a sensor array to overcome the difficulty of achieving highly specific analyte sensing in competitive solutions. Often, a single chemosensor is not enough to selectively target one analyte in a complex mixture that contains a number of structurally similar analytes and biological co-solutes. Instead of re-designing a chemosensor to improve selectivity, the original IDA can be expanded to form an array of partially promiscuous, cross-reactive chemosensors that detect many analytes but to different extents. In this approach, popularized by Anslyn and since thoroughly explored my many others, ${ }^{38}$ the output is a unique pattern or fingerprint for a given analyte that is subsequently simplified and visualized through chemometric analyses in order to distinguish different analytes (Figure 23). 
In one illustrative example that works in a real biofluid, a guanidinium-modified calix[5]arene, 31, was able to detect an ovarian cancer marker, lysophosphatidic acid (32), at nM concentrations in plasma through the displacement of fluorescein, 33 (Figure 24). ${ }^{39}$ Despite high selectivity over most other co-solutes, ATP was found to be a major competitor. An array of similar calixarenes (34) and different dyes (35) were used in combination with chemometrics to robustly distinguish ATP from the targeted biomarker, 32.

In another example, a library of dye-conjugated calix[4]pyrroles detected and discriminated between different over-the-counter carboxylate drugs in urine (Figure 25). ${ }^{40}$ Once a drug is bound to the pyrroles, the dye turns on through an intramolecular partial charge transfer. The authors initially found that each calix[4]pyrrole chemosensor bound more strongly to competitive biological anions like $\mathrm{Cl}^{-}$and $\mathrm{H}_{2} \mathrm{PO}_{4}^{-}$than with benzoate, a compound that is structurally similar to the tested over-the-counter drugs. However, the library of calix[4]pyrroles in combination of chemometrics was able to detect and successfully discriminate between the carboxylate drugs in urine.

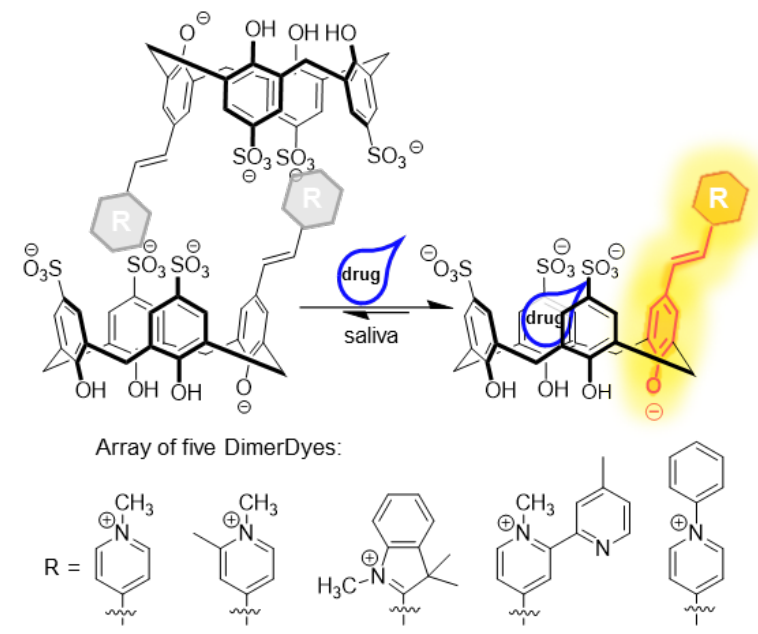

Figure 26. The DimerDye Disassembly Assay. A self-assembled, self-quenched DimerDye dissociates in the presence of hydrophobic cationic guests to yield a turn on fluorescent complex. The combination of five DimerDyes in a sensor array was able to detect and discriminate between illicit drugs and their metabolites.

An array of calix[4]arene-based chemosensors detects and discriminates between different illicit drugs in saliva, which contains $20-100 \mathrm{mM}$ of various salts and $3 \mathrm{~g} / \mathrm{L}$ of proteins. The previously mentioned salt-tolerant calixarene dimers (Figure 26) inspired the invention of chemosensors called DimerDyes that replace the pendant hydrophobic group with environment- 
sensitive merocyanine dyes. These novel chemosensors operate as a DimerDye Disassembly Assay, in which they first form self-quenched homodimers, and then operate as sensors when the homodimers disassemble in the presence of competing hydrophobic, cationic guests in order to induce a turn-on fluorescence response. To achieve successful discrimination of closely related drugs and their metabolites in human saliva, sixteen DimerDyes were made and tested in parallel, leading to the selection of five sensors that could achieve the desired sensing tasks. The combination of the selected five DimerDyes and chemometric tools were used to successfully detect and discriminate between illicit drugs such as cocaine, heroin, methamphetamine, ecstasy, and their respective metabolites at low $\mu \mathrm{M}$ concentrations in human saliva. ${ }^{41}$

\section{Hosts that operate in whole cells and cell lysates}

Some supramolecular hosts can function individually in whole cells and cell lysates, in spite of the many salts, metabolites and other biomolecules that exist in living systems. In one example, a host was used as a biomolecular enrichment tool that functioned in a cell lysate to "fish out" a protein from a complex mixture (Figure 27). ${ }^{42}$ An acetamidocalix[4]arene, 36, was conjugated to an agarose bead and mixed with HeLa cell lysate. The goal was to allow the calixarene to form complexes with a few strong protein partners from the thousands of proteins contained in the cancerous cell. "Hit" proteins were identified after a pull-down, cleavage from the bead, and proteomics analysis. Subsequent re-testing confirmed the biological activity of the calixarene on its newly discovered protein target.

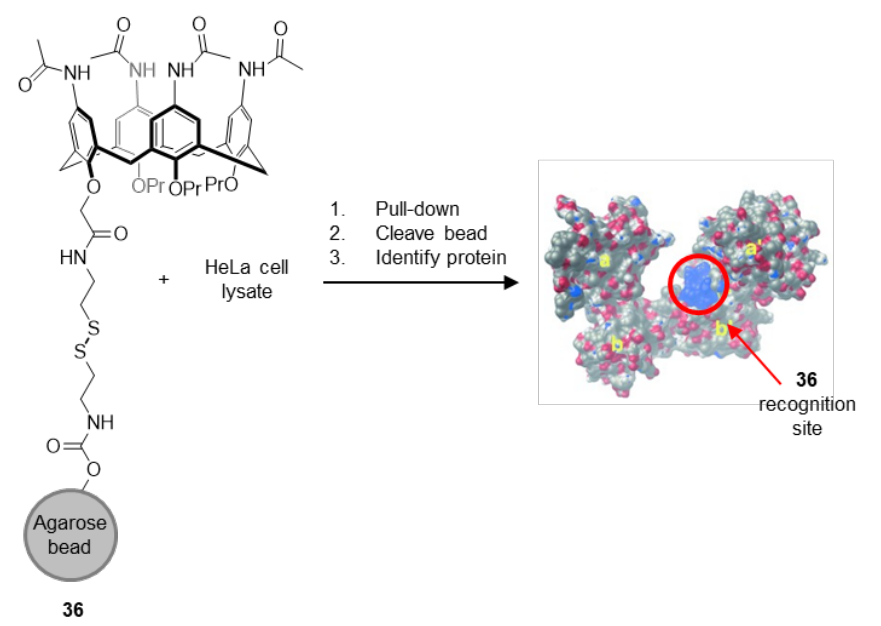

Figure 27. An unbiased approach to discovering host-guest complexes. An

acetamidocalix[4]arene (36) was conjugated to an agarose bead, and used to "fish" proteins from 
a cancer cell lysate. LC-MS/MS based proteomics analysis revealed the proteins that bound strongly. Adapted with permission from ref 42. Copyright 2015, John Wiley and Sons.
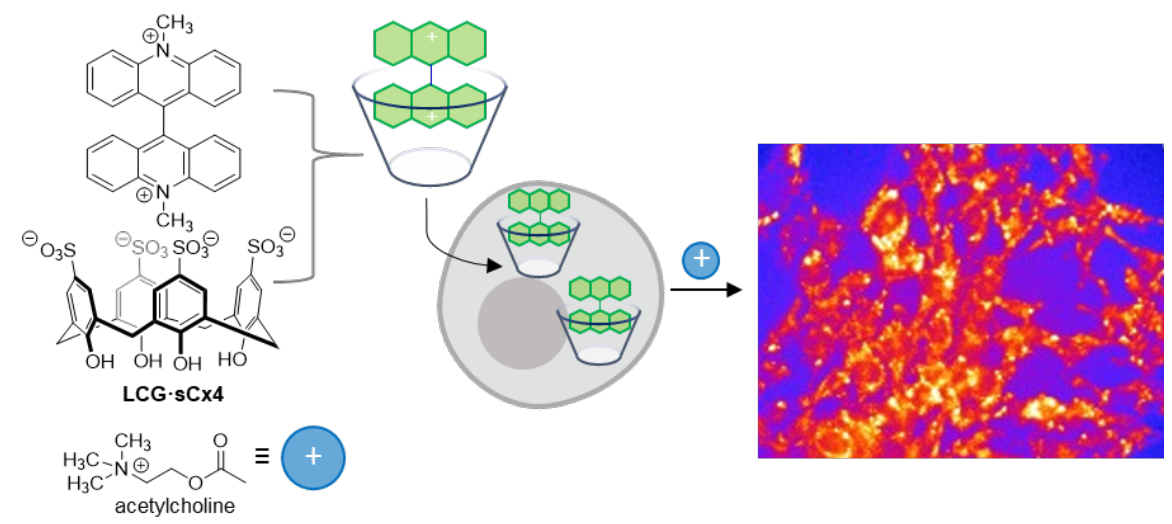

Figure 28. sCx4 and LCG enter cells and can serve as an intracellular IDA to detect acetylcholine in fibroblasts. Adapted with permission from ref 43. Copyright 2015, John Wiley and Sons.

Cells have lipid bilayers and other barriers that prevent foreign substances from entering, yet there are some supramolecular hosts that can successfully pass through membranes. While highly charged hosts often fail to enter cells, the pre-formed complex of sCx4 and lucigenin dye (LCG) penetrated and worked as an IDA sensor within live Chinese hamster ovary cells and fibroblasts (Figure 28). ${ }^{43}$ The sensing abilities were strongly affected by the chloride-containing cell medium (binding strength decreased 100-fold, quenching efficiency decreased 5-fold) yet the differential response was enough to detect guests such as acetylcholine. There are a small but growing number of such reports, where a host can enter a cell along with the guest to which it is bound. The actual mechanism behind the cellular entry of $\mathbf{s C x} 4$ remains unknown. But the ability of oppositely charged, somewhat lipophilic host-guest complexes to enter cells is reminiscent of the way that cationic lipids can bind to DNA and serve as transfection agents. 

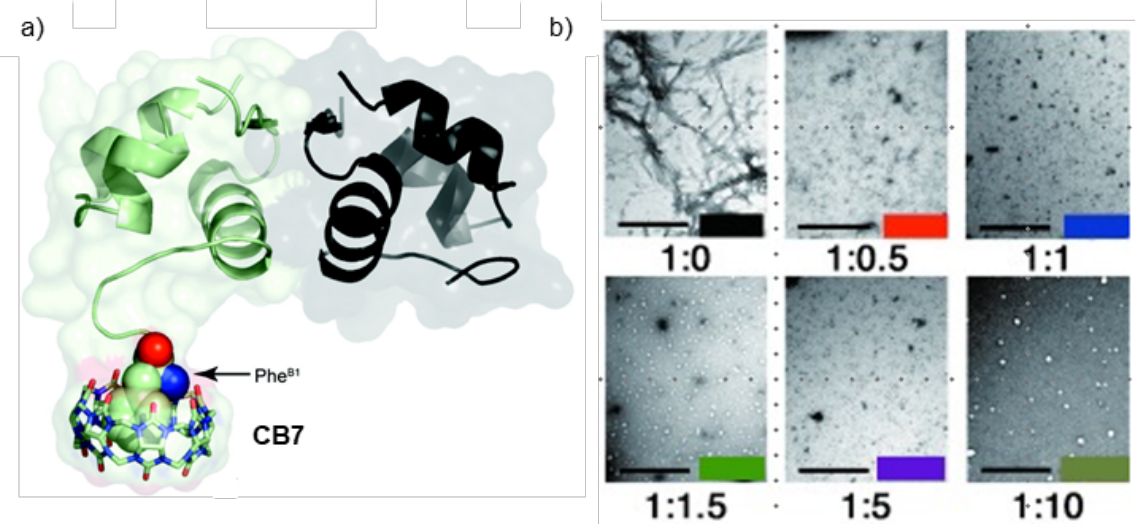

Figure 29. CB7 encapsulates the N-terminal phenylalanine on insulin, preventing toxic fibril and amyloid formation in cells. a) Crystal structure of CB7 complexed to phenylalanine derivative. Adapted with permission from ref 44. Copyright 2011, American Chemical Society. b) TEM images show a decrease of insulin fibers formed with higher ratios of CB7 (insulin:CB7 ratio). Adapted with permission from ref 45. Copyright 2014, John Wiley and Sons.

CB7s bind with $N$-terminal phenylalanine residues on proteins such as insulin with submicromolar affinities (Figure 29a). ${ }^{44}$ Many protein unfolding diseases such as Alzheimer's and amyloidosis contain hydrophobic residues, like phenylalanine, that form $\beta$-sheets which promote unfolding, refolding, and sequential formation of fibrils and amyloids. The fibrils of amyloidogenic proteins like insulin can be toxic to cells. In a promising proof-of-concept for a new kind of therapeutic approach, CB7 was able to bind insulin through its N-terminal phenylalanine residue, decrease insulin fibirillization, and reduce the toxicity of insulin fibrils to living cells in a standard cell culture medium (Figure 29b) (5 $^{45}$

\section{Supramolecular systems that function in whole organisms}
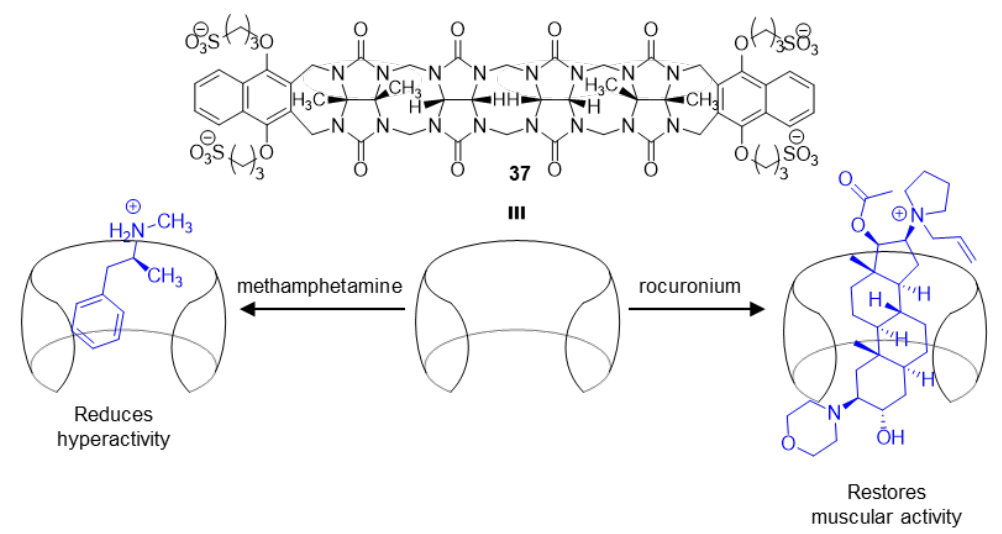

Figure 30. Acyclic cucurbituril, 37, binds to methamphetamine to reduce hyperactivity in rats and rocuronium to restore muscular activity in mice. 
For a supramolecular system to be successful in a whole organism, the host must be both tolerant of co-solutes and also behave well pharmacologically. Cucurbiturils, sCx4 and $\gamma$ cyclodextrin have been shown to be non-toxic to mice at concentrations up to $250 \mathrm{mg} / \mathrm{kg}, 100$ $\mathrm{mg} / \mathrm{kg}$, and $16,000 \mathrm{mg} / \mathrm{kg}$, respectively. ${ }^{46-48}$ These macrocycles seem to be relatively inert in the body, and examples of these hosts can act as drug carriers, bioimaging agents, disease inhibitors, and even reversal agents for other drugs.

Many examples of host-guest chemistry operating successfully in whole organisms involve supramolecular hosts acting as drug reversal agents. Many hosts including $\mathbf{s} \mathbf{C x 4}$, cyclodextrins, cyclic and acyclic cucurbiturils form strong complexes with various illicit, harmful, or toxic drugs in water with association constants ranging from $0.1 \mathrm{mM}$ to $10 \mathrm{nM} .{ }^{25}$ Compound 37 was successful at binding and removing methamphetamine in rats and reducing drug-induced hyperlocomotion. ${ }^{25}$ It was also successfully able to bind and remove rocuronium, a neuromuscular blockade agent, from mice, to restore normal muscular control after an anaesthetic procedure (Figure 30). This follows on from a currently approved cyclodextrin drug called Sugammadex, which also removes rocuronium and restores muscular activity in humans after the surgical neuromuscular blockade. ${ }^{49}$ $\mathbf{s C x} 4$ has been shown to reverse drug toxicity by, for example, lowering the concentration of a toxic herbicide, paraquat, rescuing animals in a rat model of herbicide poisoning. ${ }^{50}$ An increasing number of such examples exist for pillar[n]arenes, calix[n]arenes, CB-analogs, and cyclodextrins. An almost universal aspect of these studies is the focus on drugs (guests) that are both cationic and hydrophobic, and that are therefore well suited to being bound by anionic hosts.

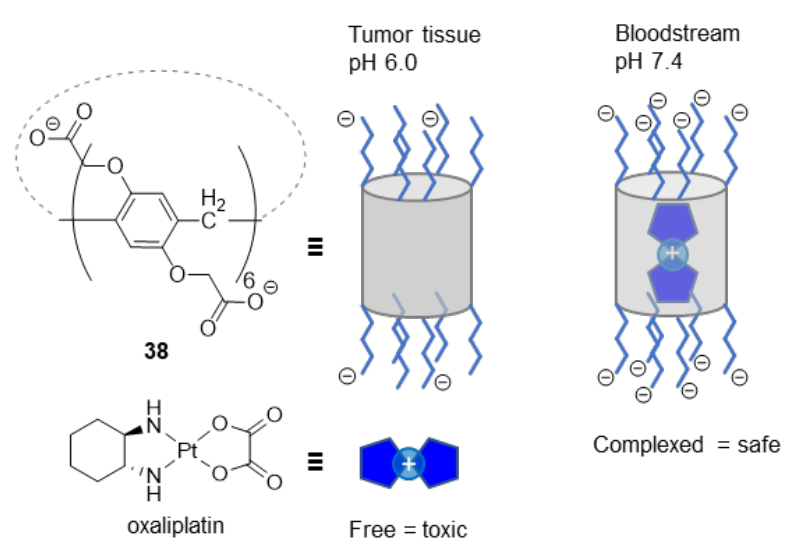

Figure 31. Carboxypillar[6]arene, 38, complexes a potent cancer drug, oxaliplatin, when deprotonated in the bloodstream but releases the drug in acidic tumour tissue. 
Hosts can also act as carriers for beneficial drugs and therapies. ${ }^{51}$ Cyclodextrins have long been used as excipients that solubilize and stabilize drugs in the bloodstream. We will present here recent examples in which a novel host releases the drug at the site of disease due to an environmental change. For example, carboxylatopillar[6]arene (38) encapsulates and transports a toxic cancer drug, oxaliplatin, to tumor cells in mice (Figure 31). ${ }^{52}$ The release of the drug is triggered by a $\mathrm{pH}$ drop in cancer tissue, causing oxaliplatin to lose affinity for protonated $\mathbf{3 8}$. In another strategy, a host can encapsulate a reactive molecule in vivo, rendering it temporarily inert. A specific biomarker localized at a diseased site can then displace the reactive guest from the host in order to kill the diseased cells. An example of this uses a modified calixarene, 39, which forms a strong complex with a photosensitizer (35) that renders it photochemically inert (Figure 32). ${ }^{53}$ The large abundance of ATP present in certain tumour cells displaces 35, and when free $\mathbf{3 5}$ is irradiated by a localized light source it creates highly toxic singlet oxygen. This approach was shown to kill tumor cells in mice with good cell-type selectivity.
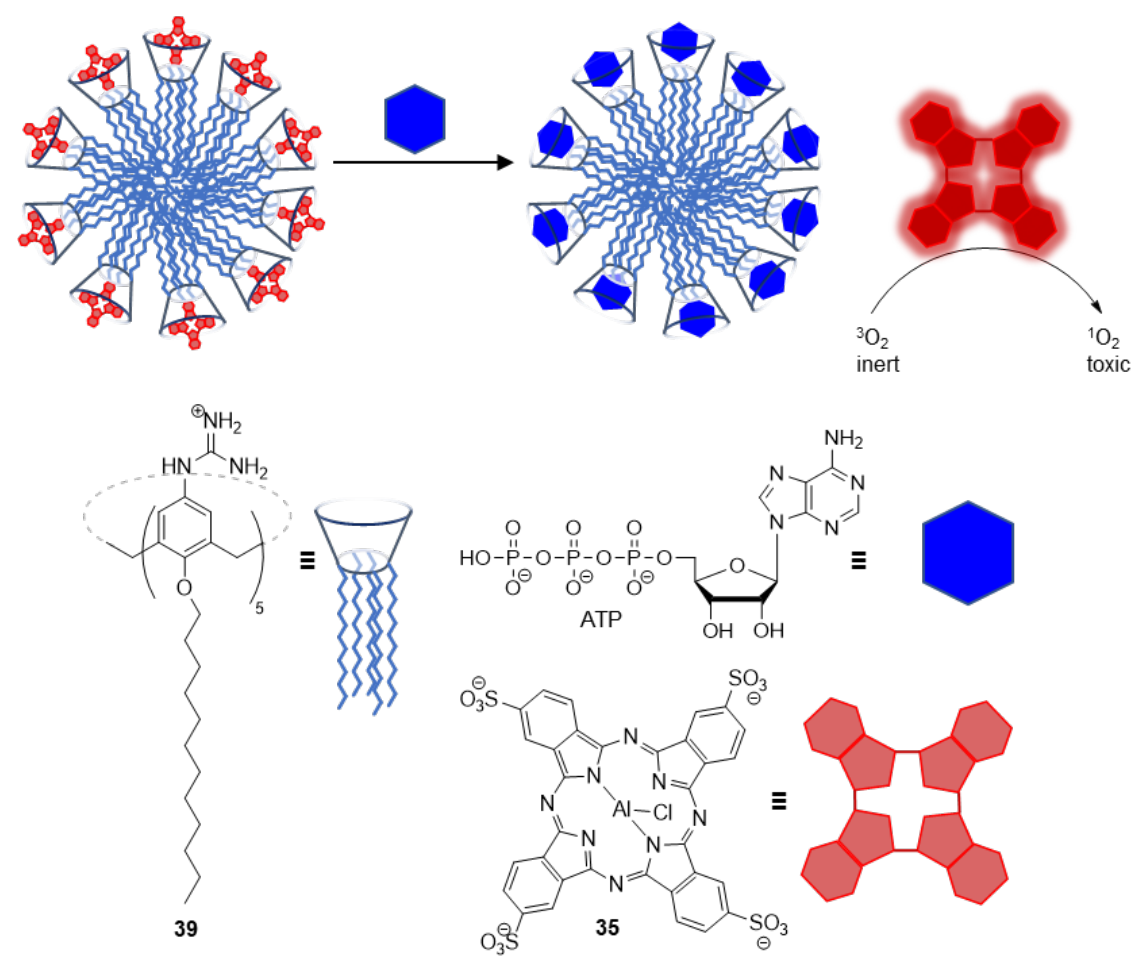

Figure 32. Biomarker displacement for phototherapy. An amphiphilic calix[5]arene, 39, binds and quenches a photosensitizer, 35, forming stable micelles that remain inert in mice. The high concentrations of ATP in tumour cells bind to the calixarene and displace 35. Irradiation of the tissues containing the now-released photosensitizer creates highly reactive singlet oxygen, selectively killing cancer cells. 


\section{Conclusion and outlook}

The field of host-guest chemistry has evolved dramatically from its origins in fundamental research. Successful complexation in water, once a frontier of discovery for the basic sciences, is now regularly achieved. The creative application of host-guest chemistry could offer solutions to diverse kinds of problems in the life sciences, and this approach is already starting to make major impacts in biomedical research and in medicine. We hope that this practically oriented tutorial review both encourages and enables newcomers to contribute to this exciting field.

\section{Acknowledgements}

We thank co-workers past and present for help in learning the difficult lessons of molecular recognition in warm, salty water. FH thanks the Canada Research Chairs program for ongoing support.

\section{Funding}

This work was supported by NSERC Discovery Grant RGPIN-2019-04806.

\section{Declaration}

The authors declare no conflict of interest.

\section{Keywords}

Host-guest chemistry, molecular recognition, water, salt effects, self-assembly, macrocycles, hydrophobic effect
Abbreviations
$\mathrm{ACh}$
acetylcholine
ATP
adenosine triphosphate 


$\begin{array}{ll}\text { aRme2 } & \text { asymmetric dimethylarginine } \\ \text { CB } & \text { cucurbituril } \\ \text { CD } & \text { cyclodextrin } \\ \text { calixarene-induced aggregation } \\ \text { dimethylsulfoxide } \\ \text { DMSO } & \text { deoxyribonucleic acid } \\ \text { DNA } & \text { indicator displacement assay } \\ \text { IDA } & \text { dissociation constant } \\ K_{d} & \text { trimethyllysine } \\ \text { Kme3 } & \text { lucigenin } \\ \text { LCG } & \text { nuclear magnetic resonance } \\ \text { NMR } & \text { statistical assessment of the modelling of protein and } \\ \text { SAMPL } & \text { ligand } \\ \text { sCx4 } & \text { para-sulfonatocalix[4]arene }\end{array}$

\section{References}

1. E. V. Anslyn and D. A. Dougherty, Modern physical organic chemistry, University Science Books, 2006, and references therein.

2. P. Neri, Jonathan L. Sessler and M.-X. W. (eds), Calixarenes and beyond, Springer, 2016, and references therein.

3. W. Saenger, Angew. Chem. Int. Ed., 1980, 19, 344-362, and references therein.

4. T. Ogoshi, S. Takashima and T.-a. Yamagishi, J. Am. Chem. Soc., 2015, 137, 1096210964.

5. W. Xue, P. Y. Zavalij and L. Isaacs, Angew. Chem. Int. Ed., 2020, 59, 13313-13319.

6. H. Zhang, X. Ma, K. T. Nguyen and Y. Zhao, ACS Nano, 2013, 7, 7853-7863.

7. K. Kim, N. Selvapalam and D. H. Oh, J. Inclusion Phenom. Macrocyclic Chem., 2004, 50, 31-36.

8. B. Vinciguerra, L. Cao, J. R. Cannon, P. Y. Zavalij, C. Fenselau and L. Isaacs, J. Am. Chem. Soc., 2012, 134, 13133-13140.

9. H.-W. Tian, Y.-C. Liu and D.-S. Guo, Mater. Chem. Front., 2020, 4, 46-98, and references therein.

10. K. Wang, D.-S. Guo, X. Wang and Y. Liu, ACS Nano, 2011, 5, 2880-2894.

11. F. Corbellini, R. Fiammengo, P. Timmerman, M. Crego-Calama, K. Versluis, A. J. R. Heck, I. Luyten and D. N. Reinhoudt, J. Am. Chem. Soc., 2002, 124, 6569-6575.

12. F. Corbellini, L. Di Costanzo, M. Crego-Calama, S. Geremia and D. N. Reinhoudt, J. Am. Chem. Soc., 2003, 125, 9946-9947.

13. Y. Hua, R. O. Ramabhadran, J. A. Karty, K. Raghavachari and A. H. Flood, Chem. Commun., 2011, 47, 5979-5981.

14. Y. Liu, W. Zhao, C.-H. Chen and A. H. Flood, Science, 2019, 365, 159-161.

15. J. Murray, K. Kim, T. Ogoshi, W. Yao and B. C. Gibb, Chem. Soc. Rev., 2017, 46, 24792496, and references therein.

16. J. C. Ma and D. A. Dougherty, Chem. Rev., 1997, 97, 1303-1324, and references therein.

17. D. A. Dougherty, Acc. Chem. Res., 2013, 46, 885-893, and references therein.

18. F. Perret, J.-P. Morel and N. Morel-Desrosiers, Supramol. Chem., 2003, 15, 199-206. 
19. K. D. Daze, C. E. Jones, B. J. Lilgert, C. S. Beshara and F. Hof, Can. J. Chem., 2013, 91, 1072-1076.

20. F. Biedermann, W. M. Nau and H. J. Schneider, Angew. Chem. Int. Ed., 2014, 53, 1115811171, and references therein.

21. S. He, F. Biedermann, N. Vankova, L. Zhechkov, T. Heine, R. E. Hoffman, A. De Simone, T. T. Duignan and W. M. Nau, Nat. Chem., 2018, 10, 1252-1257.

22. L. I. James, J. E. Beaver, N. W. Rice and M. L. Waters, J. Am. Chem. Soc., 2013, 135, 6450-6455.

23. D.-S. Guo, V. D. Uzunova, X. Su, Y. Liu and W. M. Nau, Chem. Sci., 2011, 2, 17221734.

24. N. Lavande, A. Acuña, N. Basílio, V. Francisco, D. D. Malkhede and L. Garcia-Rio, Phys. Chem. Chem. Phys., 2017, 19, 13640-13649.

25. S. Ganapati, S. D. Grabitz, S. Murkli, F. Scheffenbichler, M. I. Rudolph, P. Y. Zavalij, M. Eikermann and L. Isaacs, ChemBioChem, 2017, 18, 1583-1588.

26. V. Francisco, A. Piñeiro, W. M. Nau and L. García-Río, Chem. Eur. J., 2013, 19, 1780917820.

27. S. S. Thomas, H. Tang and C. Bohne, J. Am. Chem. Soc., 2019, 141, 9645-9654.

28. C. L. D. Gibb and B. C. Gibb, J. Am. Chem. Soc., 2011, 133, 7344-7347.

29. K. I. Assaf and W. M. Nau, Angew. Chem. Int. Ed., 2018, 57, 13968-13981.

30. N. Kon, N. Iki and S. Miyano, Org. Biomol. Chem., 2003, 1, 751-755.

31. R. N. Dsouza, U. Pischel and W. M. Nau, Chem. Rev., 2011, 111, 7941-7980, and references therein.

32. M. Florea, S. Kudithipudi, A. Rei, M. J. González-Álvarez, A. Jeltsch and W. M. Nau, Chem. Eur. J. , 2012, 18, 3521-3528.

33. K. D. Legg and D. M. Hercules, J. Phys. Chem., 1970, 74, 2114-2118.

34. Y. Liu, L. Perez, M. Mettry, C. J. Easley, R. J. Hooley and W. Zhong, J. Am. Chem. Soc., 2016, 138, 10746-10749.

35. M. B. Hillyer, H. Gan and B. C. Gibb, ChemPhysChem, 2018, 19, 2285-2289.

36. G. A. Garnett, K. D. Daze, J. A. Pena Diaz, N. Fagen, A. Shaurya, M. C. Ma, M. S. Collins, D. W. Johnson, L. N. Zakharov and F. Hof, Chem. Commun., 2016, 52, 27682771.

37. S. Bouatra, F. Aziat, R. Mandal, A. C. Guo, M. R. Wilson, C. Knox, T. C. Bjorndahl, R. Krishnamurthy, F. Saleem, P. Liu, Z. T. Dame, J. Poelzer, J. Huynh, F. S. Yallou, N. Psychogios, E. Dong, R. Bogumil, C. Roehring and D. S. Wishart, PloS One, 2013, 8, e73076-e73076.

38. S. Stewart, M. A. Ivy and E. V. Anslyn, Chem. Soc. Rev., 2014, 43, 70-84, and references therein.

39. Z. Zheng, W.-C. Geng, J. Gao, Y.-Y. Wang, H. Sun and D.-S. Guo, Chem. Sci., 2018, 9, 2087-2091.

40. Y. Liu, T. Minami, R. Nishiyabu, Z. Wang and P. Anzenbacher, J. Am. Chem. Soc., 2013, 135, 7705-7712.

41. M. A. Beatty, A. J. Selinger, Y. Li and F. Hof, J. Am. Chem. Soc., 2019, 141, 1676316771.

42. S. Tommasone, C. Talotta, C. Gaeta, L. Margarucci, M. C. Monti, A. Casapullo, B. Macchi, S. P. Prete, A. Ladeira De Araujo and P. Neri, Angew. Chem. Int. Ed., 2015, 54, 15405-15409. 
43. A. Norouzy, Z. Azizi and W. M. Nau, Angew. Chem. Int. Ed., 2015, 54, 792-795.

44. J. M. Chinai, A. B. Taylor, L. M. Ryno, N. D. Hargreaves, C. A. Morris, P. J. Hart and A. R. Urbach, J. Am. Chem. Soc., 2011, 133, 8810-8813.

45. H. H. Lee, T. S. Choi, S. J. C. Lee, J. W. Lee, J. Park, Y. H. Ko, W. J. Kim, K. Kim and H. I. Kim, Angew. Chem. Int. Ed., 2014, 53, 7461-7465.

46. A. W. Coleman, S. Jebors, S. Cecillon, P. Perret, D. Garin, D. Marti-Battle and M. Moulin, New J. Chem., 2008, 32, 780-782.

47. V. D. Uzunova, C. Cullinane, K. Brix, W. M. Nau and A. I. Day, Org. Biomol. Chem., 2010, 8, 2037-2042.

48. I. C. Munro, P. M. Newberne, V. R. Young and A. Bär, Regul. Toxicol. Pharm., 2004, 39, 3-13.

49. M. Naguib, Anesthesia \& Analgesia, 2007, 104, 575-581.

50. G.-F. Wang, X.-L. Ren, M. Zhao, X.-L. Qiu and A.-D. Qi, J. Agric. Food. Chem., 2011, 59, 4294-4299.

51. J. Zhou, G. Yu and F. Huang, Chem. Soc. Rev., 2017, 46, 7021-7053, and references therein.

52. B. Li, Z. Meng, Q. Li, X. Huang, Z. Kang, H. Dong, J. Chen, J. Sun, Y. Dong, J. Li, X. Jia, J. L. Sessler, Q. Meng and C. Li, Chem. Sci., 2017, 8, 4458-4464.

53. J. Gao, J. Li, W.-C. Geng, F.-Y. Chen, X. Duan, Z. Zheng, D. Ding and D.-S. Guo, J. Am. Chem. Soc., 2018, 140, 4945-4953. 